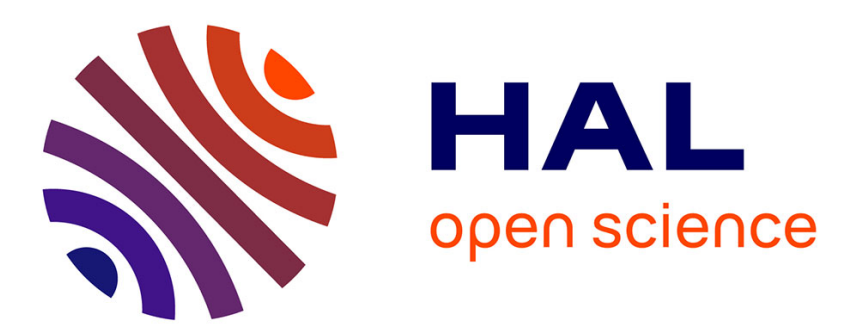

\title{
Toward an equivalence criterion for Hybrid RANS/LES methods
}

\author{
Christophe Friess, Remi Manceau, T.B. Gatski
}

\section{To cite this version:}

Christophe Friess, Remi Manceau, T.B. Gatski. Toward an equivalence criterion for Hybrid RANS/LES methods. Computers and Fluids, 2015, 122, pp.233-246. 10.1016/j.compfluid.2015.08.010 . hal-01246130

\section{HAL Id: hal-01246130 \\ https://hal.inria.fr/hal-01246130}

Submitted on 18 Dec 2015

HAL is a multi-disciplinary open access archive for the deposit and dissemination of scientific research documents, whether they are published or not. The documents may come from teaching and research institutions in France or abroad, or from public or private research centers.
L'archive ouverte pluridisciplinaire HAL, est destinée au dépôt et à la diffusion de documents scientifiques de niveau recherche, publiés ou non, émanant des établissements d'enseignement et de recherche français ou étrangers, des laboratoires publics ou privés. 


\title{
Toward an equivalence criterion for Hybrid RANS/LES methods
}

\author{
Ch. Friess ${ }^{\mathrm{a}, \mathrm{b}}$, R. Manceau ${ }^{\mathrm{a}, \mathrm{c}, *}$, T.B. Gatski ${ }^{\mathrm{a}, \mathrm{d}}$ \\ anstitute Pprime, Department of Fluid Flow, Heat Transfer and Combustion, CNRS-University of Poitiers-ENSMA, SP2MI, Bd Marie et Pierre Curie, BP \\ 30179, 86962 Futuroscope Chasseneuil Cedex, France \\ ${ }^{\mathrm{b}}$ M2P2 Lab, Aix-Marseille Université-CNRS-Ecole Centrale, 38 rue Frédéric Joliot-Curie, 13451 MARSEILLE Cedex 13 \\ ${ }^{\mathrm{c}}$ Department of Applied Mathematics, CNRS-University of Pau, and Inria-CAGIRE group, IPRA, avenue de l'université, 64013 Pau, France \\ ${ }^{\mathrm{d}}$ Center for Coastal Physical Oceanography and Ocean, Earth and Atmospheric Sciences, Old Dominion University, Norfolk, Virginia 23529 USA
}

\begin{abstract}
A criterion is established to assess the equivalence between hybrid RANS/LES methods, called H-equivalence, based on the modeled energy of the unresolved scales, which leads to similar low-order statistics of the resolved motion. Different equilibrium conditions are considered, and perturbation analyses about the equilibrium states are performed. The procedure is applied to demonstrate the equivalence between two particular hybrid methods, and leads to relationships between hybrid method parameters that control the partitioning of energy between the resolved and unresolved scales of motion. This equivalence is validated by numerical results obtained for the cases of plane and periodically constricted channel flows. This concept of H-equivalence makes it possible to view different hybrid methods as models for the same system of equations: as a consequence, detached-eddy simulation (DES), which is shown to be H-equivalent to the temporal partially integrated transport model (TPITM) in inhomogeneous, stationary situations, can be interpreted as a model for the subfilter stress involved in the temporally filtered Navier-Stokes equations.
\end{abstract}

\section{Introduction}

Numerous proposals have been made for over a decade to develop a methodology capable of bridging the large-eddy simulation (LES) and Reynolds-averaged Navier-Stokes (RANS) methodologies [1,2], in particular in a seamless way, i.e., without sharp interfaces. These bridging methodologies are intended to work simultaneously in LES mode in regions where spatially-filtered variables are desired, and in RANS mode in regions where the Reynolds averaged (statistically averaged) variables are desired. The main objective of these methods is to avoid the resolution of the whole domain in LES, since this approach is out of reach for most of the industrial applications, in particular due to the high computational cost associated with the near-wall region. Moreover, using seamless hybrid methodologies, an intermediate region necessarily exists in between the LES and RANS regions, where a limited portion of the turbulent energy is resolved. A complementary objective of these methods is then to correctly model the subgrid scales in this situation, which can be described as the very-large eddy simulation (VLES) capability of the method. Such a capability extends the flexibility of hybrid methods to the use of filters, or equivalently grid steps, larger than those used in standard LES, which can significantly reduce the computational cost. However, if the scale separation among resolved and unresolved motions lies in the productive region of the turbulent spectrum, the subgrid scale model is expected to reproduce, in addition to the transfer of energy toward the subgrid scales, complex effects of anisotropic production and redistribution. Therefore, the modeling of the subgrid-scale tensor in a VLES situation shares many features with standard RANS modeling, and, in particular, can potentially require the use of models more complex than linear-eddy viscosity models, ranging from explicit algebraic stress models $[3,4]$ to second moment closure $[5,6,7]$. The development of such models require the knowledge of the physical role of the unclosed terms, in order to guide the choice of their functional form and of the associated turbulent scales, as well as to make possible a comparison with experimental or direct numerical simulation (DNS) results.

However, in the frame of inhomogeneous turbulent flows, a trait, common among most of the hybrid RANS/LES approaches proposed so far, is the inherent difference between variables obtained from spatially filtered instantaneous variables and variables obtained from long-time averaged instantaneous variables, with the latter being representative of an ensemble averaged variable through an ergodic hypothesis [8]. This problem is a strong limitation to the understanding and modeling of the physical interaction mechanisms in the intermediate regions that are not clearly RANS or LES regions, and consequently suffer from an ambiguous defini-

\footnotetext{
*Corresponding author: remi.manceau@univ-pau.fr
} 
tion of the variables of the model. This is the reason why Spalart [9], among the future issues to be addressed in DES and, in general, hybrid RANS/LES approaches, put forth the necessity of clarifying the underlying formalism:

"The link between the DES flow field and the exact or DNS flow field should be established."

As shown in Fadai-Ghotbi et al. [10], this problem can be remedied within the context of temporal filtering, where for inhomogeneous, stationary flows, temporally-filtered quantities tend to Reynolds-averaged (long-time-averaged) quantities in the limit of infinite temporal filter width. It is worth emphasizing that, although the extension of the formalism to inhomogeneous flows is limited to stationary configurations, the practical application of the models based on this formalism is not restricted: for transient or statistically periodic flows, frequently encountered in industrial applications, such as turbomachines or wind turbines, the theoretical link with the instantaneous Navier-Stokes equations cannot be rigorously maintained but, similar to empirical approaches, the models based on temporal filtering remain applicable and relevant.

In the present work, the migration from a LES to a RANS model controlled by variable coefficients in the systems of equations for the ensemble-averaged filtered variables is investigated through a perturbation analysis about an equilibrium state. This is shown to establish an equivalence criterion between different hybrid methods, in such a way that they can be interpreted as variants of the same approach. Two hybrid methods satisfying this equivalence criterion are called herein $H$-equivalent, where $H$ stands for Hybrid. The underlying objective is to propose a possible answer to the above-mentioned difficulty to establish a link between the resolved and the exact flow fields: if an empirically-built hybrid method is H-equivalent to a method derived to close the equations obtained within a consistent formalism, it can be interpreted as a model for the same equations. To make an analogy with mathematical concepts of the set theory, the criterion provides an equivalence relation that defines an equivalence class of closure models for a given system of equations. All the models belonging to the same class can then be compared to experimental or DNS fields using the same formalism.

Such an equivalence between three hybrid methods, based on different modifications of the same underlying RANS model, was observed by Kubacki and Dick [11] when computing impinging jet cases. The equivalence criterion proposed herein provides an analytical basis for such comparisons, that can be applied to hybrid methods resting on modifications of the length and/or time scales involved in RANS models, based on dimensional arguments. Here, the procedure is applied to two distinctly formulated approaches: detached eddy simulation (DES), which is, in a variety of versions, the most widespread hybrid RANS-LES approach, and is based on an empirical modification of the dissipation rate in the underlying RANS model, in order to reduce the level of eddy viscosity and thus promote the appearance of resolved fluctuations; and partially integrated transport model (PITM), which has the highly desirable property of being based on a clear formalism and precise hypotheses to close the equations in spectral space [12]. A by-product of the present study is a new hybrid RANS/LES method that takes advantage of both the theoretical justification of the PITM and the robustness and ease of use of the DES methodology.

The paper is organized as follows: basic concepts, definitions and hypotheses, related to $\mathrm{H}$-equivalence, are set in Sec. 2. The analysis is then performed in Sec. 3, assessing an equivalence criterion between PITM and DES under various assumptions and establishing a link between the grid size and the energy partitioning between resolved and unresolved scales. The equivalence criterion is then validated in Sec. 4 upon channel flow computations: plane then periodically constricted. Finally, conclusions are drawn in Sec. 5.

\section{Definitions and postulate}

Seamless, or continuous, hybrid RANS/LES methods are usually based on modifications of the constitutive relation for the unresolved stress and/or the corresponding transport equations that are formulated within the RANS framework. These modifications aim at controlling the energy partition among the resolved and unresolved scales, and then facilitate the appearance of large-scale structures in the simulated resolved field. Although hybrid approaches are based on modifications of different terms in the systems of equations of the models, using various criteria to control the energy partition and thus the migration from RANS to LES, it can be shown, within some constraints, that an equivalence criterion can be established. The present section defines the context of the subsequent analysis.

\subsection{Self-consistent hybrid methods}

At the outset, it is useful to introduce the notion of selfconsistency, which is used in the subsequent analysis: hybrid methods are self-consistent when they replicate the behavior expected of a filtered approach, as illustrated by figure 1(a). Specifically, in hybrid methods, the migration from a RANS to a LES behavior is enforced by some control parameters introduced in the model: in principle, this migration is linked to a variation of the filter width, which modifies the partition of energy among resolved and modeled scales. As long as the cutoff wavenumber or frequency of the filter is in the inertial range of the energy spectrum, resolved dissipation remains negligible compared to modeled dissipation, such that the latter is not affected by the variation of the control parameters. Although most of the hybrid methods are empirically built by modifying the turbulent scales in a RANS model, 

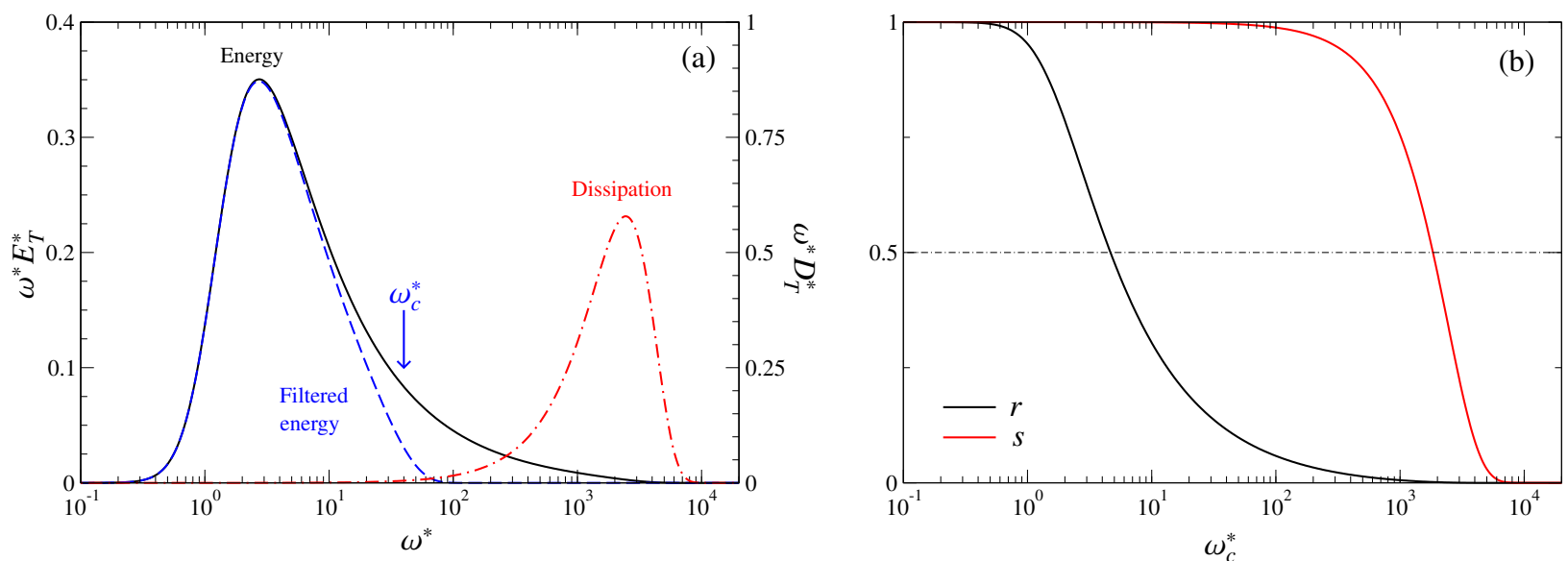

Figure 1: (a) Schematic Karman energy spectrum and associated dissipation spectrum. Stars $\left(^{*}\right)$ indicate that the quantities are expressed in units based on $k$ and $\varepsilon$. The filtered energy is evaluated using a Gaussian filter. (b) Energy ratio $r$ (ratio of subfilter to total turbulent energy, as defined by Eq. 5) and subgrid-activity parameter $s$ (ratio of subfilter to total dissipation, as defined by Eq. 4) as a function of the cutoff frequency, evaluated from this Karman spectrum.

and are not explicitly based on a filtered equations, it is desirable that they satisfy this behavior. In this case, they are qualified as self-consistent.

As shown by Gatski et al. [8], a framework can be established for self-consistent hybrid RANS-LES methodologies by defining the general filtering operator

$\tilde{f}(\mathbf{x}, t)=\int \mathcal{G}\left(\mathbf{x}, \mathbf{x}^{\prime}, t, t^{\prime}\right) f\left(\mathbf{x}^{\prime}, t^{\prime}\right) \mathrm{d} \mathbf{x}^{\prime} \mathrm{d} t^{\prime}$.

This definition introduces a filtering in both space and time, such that it encompasses various situations and solution methodologies. Standard LES methods are recovered in the case of a kernel of the form

$\mathcal{G}\left(\mathbf{x}, \mathbf{x}^{\prime}, t, t^{\prime}\right)=G\left(\mathbf{x}, \mathbf{x}^{\prime}\right) \delta\left(t^{\prime}-t\right)$,

where $G$ stands for any standard spatial filter kernel and $\delta$ is the Dirac delta function. Such a spatial framework easily provides a formalism for hybrid RANS/LES methods in the case of homogeneous turbulence, since Reynolds averaging is then equivalent to spatial averaging, i.e., to spatial filtering in the limit of infinite filter widths. In the more general and useful case of inhomogeneous, stationary turbulence, in order to formulate a seamless hybrid methodology, a time filtering operator can be defined [8], with a filter kernel of the form

$\mathcal{G}\left(\mathbf{x}, \mathbf{x}^{\prime}, t, t^{\prime}\right)=\delta_{\text {ref }}\left(\mathbf{x}^{\prime}-\mathbf{x}\right) G\left(\mathbf{x}, t^{\prime}-t\right)$,

where $G$ is a temporal filter kernel with a filter width dependent on $\mathbf{x}$, and $\delta_{\text {ref }}$ is the delta function moving with a reference velocity $\mathbf{U}_{\text {ref. }}$. In order to unambiguously define the filtering operator, the arbitrary velocity vector $\mathbf{U}_{\text {ref }}$ must be set in the observer's reference frame, and follows the usual transformation rules for velocities in a change of reference frame. With definition (3), the filter satisfies Galilean invariance, as shown by Fadai-Ghotbi et al. [10]. It is worth emphasizing that this formalism also encompasses the URANS approach, since the URANS equations can be obtained as a subset of the general equations for temporally filtered hybrid methods in the case of a temporal filter width equal to the integral time scale [10].

Within this context, the transition from RANS to LES corresponds to a variation of location of the cutoff wavenumber (spatial) or frequency (temporal), modifying the energy partition. Consequently, as long as the cutoff does not lie in the dissipative range of the spectrum, the modeled dissipation rate is not affected by this transition.

\subsection{Energy ratio $r$}

A consequence of this characteristic of self-consistent hybrid methods is that, in the budget of the resolved kinetic energy, the molecular dissipation rate $\varepsilon_{\mathrm{R}}=v \overline{\tilde{S}_{i j} \tilde{S}_{i j}}$, where $\tilde{S}_{i j}$ denotes the filtered strain tensor, an overbar the ensemble average and $v$ the molecular viscosity, remains negligible compared to the so-called turbulent dissipation $-\overline{\tau_{i j_{\mathrm{SFS}}} \tilde{S}_{i j}}$ [13], where $\tau_{i j_{\mathrm{SFs}}}$ denotes the subfilter (SFS) stress tensor. Since $-\overline{\tau_{i j_{\mathrm{SFS}}} \tilde{S}_{i j}}$ is also the rate of production of subfilter energy, it is herein denoted by $P_{M}$. The subgrid activity $p a-$ rameter $s$, which represents the portion of the dissipation of resolved energy due to the subfilter-scale model,

$s=\frac{P_{\mathrm{M}}}{P_{\mathrm{M}}+\varepsilon_{\mathrm{R}}}$,

as defined by Geurts and Fröhlich [13], thus remains close to unity. As illustrated by Fig. 1(b), s is the relevant parameter to identify the resolution of LES as compared to a well-resolved DNS, and the criterion $s=0.5$ retained by Geurts and Fröhlich obviously corresponds to a cutoff frequency lying in the dissipative range. In the framework of hybrid RANS/LES methods, in the region of transition from a RANS to a LES behavior, $s$ will remain very close to unity, 
such that a better parameter to characterize this transition is the energy ratio

$r=\frac{k_{\mathrm{M}}}{k_{\mathrm{R}}+k_{\mathrm{M}}}=\frac{k_{\mathrm{M}}}{k}$

where $k_{\mathrm{M}}=\frac{1}{2} \overline{\tau_{i i \mathrm{sFs}}}$ and $k_{\mathrm{R}}=\frac{1}{2} \overline{\tilde{u}_{i} \tilde{u}_{i}}$ are the subfilter (modeled) and resolved turbulent energy, respectively. As shown in Fig. 1(b), this ratio approaches unity for a nondimensional cutoff frequency close to unity, i.e., corresponding to the characteristic frequency of the largest turbulent scales (RANS/URANS limit); reaches the threshold $r=0.5$ in the energetic range of the spectrum; approaches zero for a well-resolved LES (Pope's definition [14] of LES corresponds to $r<0.2$ ); and reaches $r=0$ for DNS. $r$ is therefore a relevant parameter to identify the transition from a RANS to a LES behavior of the hybrid RANS/LES methods.

\subsection{H-equivalence}

A basis of comparison now needs to be defined between hybrid methods. The effects of any other characterizing features of each method should be minimized so that this comparison basis be effective in establishing a meaningful equivalence relationship. Each method being evaluated should be based on the same closure, that is, even though they use a different way to control the migration from RANS to LES, the constitutive relation for the unresolved turbulent stress field and the associated scale-providing transport equations should be identical at the RANS limit. Within this constraint, hybrid approaches can be said to use the same closure, but a different hybridization method.

When the control parameters are adjusted so that the level of modeled energy is the same for two methods, their respective resolved fields will be statistically similar. This statistical similarity includes the mean and second-order moments as well as associated correlations. Obviously, the instantaneous, resolved fields given by the two methods will differ and a similarity of their high-order statistics is not expected. However, as soon as they are intended to represent the same scales, i.e., the partition of energy is the same, and they are based on the same closure to represent the unresolved turbulent stresses, statistics such as the mean flow and the second moments will not differ significantly. This is not a very restrictive assumption, since, for two hybrid approaches based on the same closure, their systems of equations only differ by the turbulent scales made variable in order to hybridize RANS and LES, such that, if the scales are rigorously identical, the equations are rigorously the same. Therefore, two approaches based on the same closure and leading to the same energy partition in a particular situation will be called H-equivalent.
In summary, we put forth the following postulate:

Postulate: Two hybrid approaches based on the same closure, but using a different method of control of the energy partition, yield similar low-order statistics of the resolved velocity fields provided that they yield the same level of subfilter energy.

Once this postulate is admitted, the analysis can focus on the link between the form of the system of equations and the level of subfilter energy. Since the equivalence property is not concerned with the instantaneous field, but rather with the ensemble-averaged level of subfilter energy, the following analysis focuses on the equations for the ensembleaveraged filtered variables.

\section{Application of the Criterion}

\subsection{Description of the hybrid methods}

The equivalence criterion can be applied to hybrid methods with very diverse formulation developments. The hybridization method can be based on various ways of modifying the scales involved in the equations: in the eddy-viscosity algebraic relation [15, 16] or its transport equation [17], in the turbulent kinetic energy equation $[18,19]$, or in the scale providing equation [20,21,22], or any combination of these methods $[23,11]$. In order to illustrate the procedure that can be followed and the conclusions that can be drawn from the application of the criterion, two particular hybrid methods are selected, the partially integrated transport model [20,5] (PITM) and the two-equation detached eddy simulation [18] (DES) model.

The PITM method can be applied to any RANS model to derive an hybrid RANS-LES model. It is based on transport equations for the spectrally partitioned turbulent kinetic energy [20] or full Reynolds stress transport equations [5]. Additionally, the PITM methodology has been adapted to within a temporal filtering framework, that is T-PITM [10], so that for inhomogeneous, stationary flows, in the limit of infinite (temporal) filter width, the filtered equations are consistent with the long-time averaged (RANS) equations. In the spatial [20,24] (resp., temporal [10]) filtering context, it was shown that, by partially integrating (hence the name PITM) in the wavenumber (resp., frequency) space the transport equations for the energy spectrum, the transport equations for the ensemble-averaged quantities can be derived. The transport equation for the ensemble-averaged subfilter stress tensor $\tau_{i j_{\mathrm{M}}}=\overline{\tau_{i j_{\mathrm{SFS}}}}$ is

$\frac{D \tau_{i j_{\mathrm{M}}}}{D t}=P_{i j_{\mathrm{M}}}+\Phi_{i j_{\mathrm{M}}}-\varepsilon_{i j_{\mathrm{M}}}+D_{i j_{\mathrm{M}}}$.

Here, it is worth emphasizing that, although the PITM solves the time-dependent, filtered equations of motion (see section 4 ), the analysis is, as usual [5,24,10], performed in a statistical framework, in order to analyze how the different terms 
depend on the filter width. Therefore, in the present section, we are concerned with ensemble-averaged filtered quantities and their equations: in homogeneous situations, the material derivative $D / D t$ reduces to a time derivative $\mathrm{d} / \mathrm{d} t$, since the ensemble-averaged filtered variables are functions of $t$ only, i.e., $\overline{\widetilde{\Phi}(\mathbf{x}, t)}=\Phi_{\mathrm{M}}(t)$, for any filtered variable $\widetilde{\Phi}$; in contrast, in inhomogeneous, stationary turbulence, the material derivative is $U_{k} \partial / \partial x_{k}$, where $U_{k}$ stands for the mean velocity, since the ensemble-averaged filtered variables are functions of $\mathbf{x}$ only, i.e., $\overline{\widetilde{\Phi}(\mathbf{x}, t)}=\Phi_{\mathrm{M}}(\mathbf{x}) . P_{i j_{\mathrm{M}}}, \Phi_{i j_{\mathrm{M}}}, D_{i j_{\mathrm{M}}}$ and $\varepsilon_{i j_{\mathrm{M}}}$ represent production of subfilter stresses, redistribution, diffusion and dissipation, respectively (for details, see Chaouat and Schiestel [24, 12]). Although the approach is generally used with a second moment closure, i.e., the transport equations (6) for the subfilter stresses are used, the analysis herein focuses on the behavior of the subfilter or modeled energy $k_{\mathrm{M}}=\frac{1}{2} \overline{\tau_{i i_{\mathrm{SF}}}}$, such that the transport equation for the latter is required, as well as that of its dissipation rate $\varepsilon_{\mathrm{M}}$ :

$\frac{D k_{\mathrm{M}}}{D t}=P_{\mathrm{M}}-\varepsilon_{\mathrm{M}}+D_{\mathrm{M}}$,

$\frac{D \varepsilon_{\mathrm{M}}}{D t}=C_{\varepsilon 1} \frac{\varepsilon_{\mathrm{M}}}{k_{\mathrm{M}}} P_{\mathrm{M}}-C_{\varepsilon 2}^{*} \frac{\varepsilon_{\mathrm{M}}^{2}}{k_{\mathrm{M}}}+D_{\varepsilon}$,

where $P_{\mathrm{M}}=\frac{1}{2} P_{i j_{\mathrm{M}}}, \varepsilon_{\mathrm{M}}=\frac{1}{2} \varepsilon_{i j_{\mathrm{M}}}$ and $D_{\mathrm{M}}=\frac{1}{2} D_{i j_{\mathrm{M}}}$ are production, dissipation and diffusion rates, respectively. These equations are formally similar to the RANS equations. The dissipation equation is sensitized to the variable wavenumber (resp., frequency) cutoff by using a variable coefficient for the destruction term

$C_{\varepsilon 2}^{*}=C_{\varepsilon_{1}}+r\left(C_{\varepsilon_{2}}-C_{\varepsilon_{1}}\right)$.

Note that PANS [21], albeit based on a completely different argumentation, also uses Eqs. (7), (8) and (9). The most recent version of PANS [25, 26] uses relation (49) between $r$ and the grid step, such as it is H-equivalent to PITM in the equilibrium layers considered in section 3.2 and only differ by the diffusion coefficients in inhomogeneous flows.

In the PITM approach, the fundamental parameter is thus the ratio of modeled energy $k_{\mathrm{M}}$ to total energy $k$ defined by Eq. (5). Although the formalism is different in PITM and T-PITM (spatial filtering vs. temporal filtering) and, thus, the variables do not represent the same physical quantities, the form of the system of equations is the same. Since the method used below to establish the equivalence between hybrid methods is only based on the analysis of the properties of the system of equations, the distinction between PITM and T-PITM will not be necessary in the rest of section 3, and the two methods are simply denoted by PITM.

In the following sections, the analysis focuses on the $\mathrm{H}$ equivalence between the PITM and DES approaches. DES was originally [17] based on an empirical modification of the length-scale entering the dissipation term in the SpalartAllmaras model, with the objective of allowing the model to migrate from a RANS behavior in attached boundary layers to a LES behavior in detached regions, by reducing the amount of eddy viscosity in the momentum equation. It can be generalized to any two-equation model [18] or secondmoment closure by modifying the length scale in the dissipation term of the kinetic energy (or Reynolds stress) transport equation. Other variations of this method have emerged since its inception [9]; however, for the comparative purposes here, the baseline form will highlight the steps necessary in establishing method equivalence.

In DES based on two-equation models (resp., second moment closures), the RANS-LES hybridization is performed through a modification of the dissipation term in the transport equation for the subfilter kinetic energy (resp., the subfilter stress tensor). In order to establish the H-equivalence of the PITM and DES approaches, the system of Eqs. (6)-(8) is empirically reformulated in the DES form

$$
\begin{aligned}
& \frac{D \tau_{i j_{\mathrm{M}}}}{D t}=P_{i j_{\mathrm{M}}}+\Phi_{i j_{\mathrm{M}}}-\psi \varepsilon_{i j_{\mathrm{M}}}+D_{i j_{\mathrm{M}}}, \\
& \frac{D k_{\mathrm{M}}}{D t}=P_{\mathrm{M}}-\psi \varepsilon_{\mathrm{M}}+D_{\mathrm{M}}, \\
& \frac{D \varepsilon_{\mathrm{M}}}{D t}=C_{\varepsilon 1} \frac{\varepsilon_{\mathrm{M}}}{k_{\mathrm{M}}} P_{\mathrm{M}}-C_{\varepsilon 2} \frac{\varepsilon_{\mathrm{M}}^{2}}{k_{\mathrm{M}}}+D_{\varepsilon} .
\end{aligned}
$$

In comparison to the PITM system (6)-(8), the DES system uses a constant coefficient $C_{\varepsilon 2}$ in the dissipation equation and a variable coefficient $\psi$ in the subfilter stress or the subfilter energy equation. Again, Eq. (10) will not be used in the analysis.

In order for the system to represent both the PITM and DES methods, the transport equations for the modeled energy and its dissipation rate can be generalized as

$$
\begin{aligned}
& \frac{D k_{\mathrm{M}}}{D t}=P_{\mathrm{M}}-\psi \varepsilon_{\mathrm{M}}+D_{\mathrm{M}}, \\
& \frac{D \varepsilon_{\mathrm{M}}}{D t}=C_{\varepsilon 1} \frac{\varepsilon_{\mathrm{M}}}{k_{\mathrm{M}}} P_{\mathrm{M}}-C_{\varepsilon 2}^{*} \frac{\varepsilon_{\mathrm{M}}^{2}}{k_{\mathrm{M}}}+D_{\varepsilon} .
\end{aligned}
$$

For PITM, $\psi=1$ and $C_{\varepsilon 2}^{*}$ is given by Eq. (9), and for DES, $C_{\varepsilon 2}^{*}=C_{\varepsilon 2}$ and $\psi$ is given by

$\psi=\max \left(1 ; \frac{k_{\mathrm{M}}^{3 / 2} / \varepsilon_{\mathrm{M}}}{C_{\mathrm{DES}} \Delta}\right)$,

with $\Delta$ the grid step and $C_{\mathrm{DES}}$ a calibration coefficient. The particular system (13)-(14) is adequate to compare the PITM and DES methods: the same procedure can be applied to different systems of equations for different choices of hybrid methods. Again, it is worth pointing out that these equations are valid for eddy-viscosity models as well as for second moment closures, since Eq. (13) can be obtained by contracting the subfilter-stress transport equations. Note that for the present purpose, near-wall, low Reynolds number regions, treated in RANS mode, do not need to be considered, so 
that the additional complexity introduced by near-wall corrections is avoided.

In the system (13)-(14),

$P_{\mathrm{M}}=\overline{\left(P_{\mathrm{SFS}}\right)}=-\overline{\tau_{i j \mathrm{sFS}} \tilde{S}_{i j}}=-\overline{k_{\mathrm{SFS}} b_{i j \mathrm{sFs}} \tilde{S}_{i j}}$,

where $b_{i j \mathrm{sFs}}=\tau_{i j \mathrm{sFs}} /\left(2 k_{\mathrm{sFs}}\right)-\delta_{i j} / 3$ is the subfilter anisotropy, such that it is natural to rescale it using $S=\sqrt{2 \overline{\tilde{S}_{i j} \tilde{S}_{i j}}}$ and $k_{\mathrm{M}}$, thus defining

$\gamma=\frac{P_{\mathrm{M}}}{k_{\mathrm{M}} S}=\frac{\overline{k_{\mathrm{sFs}} b_{i j \mathrm{sFs}} \tilde{S}_{i j}}}{k_{\mathrm{M}} S}$,

which is merely a generalized correlation coefficient.

Note that Eqs. (13) and (14) only describe the evolution of the ensemble-averaged subfilter energy and dissipation, $k_{\mathrm{M}}$ and $\varepsilon_{\mathrm{M}}$ : as each method uses a different way of partitioning resolved and unresolved energy, local and instantaneous dynamics may differ significantly between each other. This is why the equivalence can only be stated on low-order statistics of the flow, as precised in the postulate of Section 2. Despite the fact that, as mentioned by Spalart [9], a fully satisfactory formalism is not available for DES, it implicitly evolved from a spatially filtered framework, so that only for homogeneous flows are the statistically-averaged fields equivalent to spatially-filtered fields in the limit of infinite filter width. Although each method, DES and PITM, have diverse origins, it will be shown that an equivalence can be established, following the definition given in Sec. 2, and that the energy partitioning parameters can be related. A comprehensive way of posing the problem of $\mathrm{H}$-equivalence is the question: in the system (11)-(12), what $\psi$ should be imposed, instead of Eq. (15), to reach the same mean equilibrium as the system (7)-(8) for a given $C_{\varepsilon 2}^{*}$ provided by the PITM theory? With the specification of the methods complete, it is now possible, in simplified situations, to establish the H-equivalence between the models and to identify the key equivalence parameters, for two families of flows.

\subsection{H-equivalence in equilibrium layers}

To first illustrate how the H-equivalence of the hybrid models can be determined, consider simple situations, such as homogeneous shear or in the logarithmic region of a boundary layer. In these cases, the turbulence evolves to a state where equilibrium values are reached for quantities such as the production-to-dissipation ratio, time scale, and turbulent stress anisotropies. Since H-equivalence is based on energy partitioning, the analysis focuses on variations of modeled kinetic energy. This variation is controlled by the parameters $\psi$ and $C_{\varepsilon 2}^{*}$. Now, one aims at modifying the energy partition in the flow domain by applying either a variation $\delta \psi$ of $\psi$ or a variation $\delta C_{\varepsilon 2}^{*}$ of $C_{\varepsilon 2}^{*}$. With the objective of a perturbation analysis, the variation $\delta k_{\mathrm{M}}$ of the modeled energy $k_{\mathrm{M}}$ is a given infinitesimal percentage, i.e., the relative variation $\delta k_{\mathrm{M}} / k_{\mathrm{M}}$ is imposed and constant throughout the domain (in the perturbation analyses performed in the present article, the relative variation of any quantity $\Phi$ denotes the ratio of the infinitesimal variation $\delta \Phi$ to the initial value of this quantity, i.e., $\delta \Phi / \Phi)$. In order to know which $\delta \psi$ or $\delta C_{\varepsilon 2}^{*}$ is to be applied, a relation must be found between $\delta k_{\mathrm{M}}$ and $\delta \psi$ or $\delta C_{\varepsilon 2}^{*}$.

In equilibrium layers, the turbulent time scale $\tau=k_{\mathrm{M}} / \varepsilon_{\mathrm{M}}$ asymptotically tends to equilibrium, $D \tau / D t=0$, such that, from Eqs. (13) and (14), an equation for $\tau$ can be written as

$\left(C_{\varepsilon 1}-1\right) \frac{P_{\mathrm{M}}}{\varepsilon_{\mathrm{M}}}+\frac{k_{\mathrm{M}}}{\varepsilon_{\mathrm{M}}^{2}} D_{\varepsilon}+\left(\psi-C_{\varepsilon 2}^{*}-\frac{D_{\mathrm{M}}}{\varepsilon_{\mathrm{M}}}\right)=0$,

where, due to global or local homogeneity, $D_{\mathrm{M}}=D_{\varepsilon}=0$, leading to the equilibrium solution

$\frac{\gamma\left(C_{\varepsilon 1}-1\right)}{C_{\varepsilon 2}^{*}-\psi} \frac{S k_{\mathrm{M}}}{\varepsilon_{\mathrm{M}}}=1$,

in which Eq. (17) has been used. Consequently, for PITM, using $\psi=1$ and introducing in Eq. (19) infinitesimal perturbations $\delta \gamma, \delta S, \delta k_{\mathrm{M}}$ and $\delta C_{\varepsilon 2}^{*}$ of the variables, it is simple algebra to obtain the relation

$\delta C_{\varepsilon 2}^{*}=\left(C_{\varepsilon 2}^{*}-1\right)\left(\frac{\delta k_{\mathrm{M}}}{k_{\mathrm{M}}}+\frac{\delta \gamma}{\gamma}+\frac{\delta S}{S}\right)$,

which shows that a decrease of the amount of modeled energy (migration towards LES mode) is linked to a reduction of the $C_{\varepsilon 2}^{*}$ coefficient (note that the PITM theory $[5,20]$ shows that $C_{\varepsilon 2}^{*}$ is in the range $\left[C_{\varepsilon 1} ; C_{\varepsilon 2}\right]$, such that the factor $\left(C_{\varepsilon 2}^{*}-1\right)$ is always positive). In this derivation, the selfconsistency of the hybrid method is assumed, such that the dissipation rate is not affected by the modification of the energy partition $\left(\delta \varepsilon_{\mathrm{M}}=0\right)$. In the case of the DES system of equations, $C_{\varepsilon 2}^{*}=C_{\varepsilon 2}$ and $\delta \psi \neq 1$, and the same perturbation analysis yields

$\delta \psi=-\left(C_{\varepsilon 2}-\psi\right)\left(\frac{\delta k_{\mathrm{M}}}{k_{\mathrm{M}}}+\frac{\delta \gamma}{\gamma}+\frac{\delta S}{S}\right)$.

Since it will be shown (see Eq. 23) that $\psi$ is in the range $\left[1 ; 1+C_{\varepsilon 2}-C_{\varepsilon 1}\right]$, the factor $\left(C_{\varepsilon 2}-\psi\right)$ is positive, such that a decrease of $k_{M}$ corresponds to an increase of $\psi$.

Applying the postulate presented in Section 2, that is, the same modification $\delta k_{\mathrm{M}}$ of the modeled energy for the two methods, PITM and DES, corresponds to the same modification of the low-order statistics of the resolved scales $\delta S$ and $\delta \gamma$, then if the two methods are H-equivalent for some initial values of $C_{\varepsilon 2}^{*}$ and $\psi$, they remain equivalent, according to (20) and (21), if the modifications of their control coefficients are related by

$\frac{\delta C_{\varepsilon 2}^{*}}{C_{\varepsilon 2}^{*}-1}=-\frac{\delta \psi}{C_{\varepsilon 2}-\psi}$.

Now, there is at least one initial state for which the two methods are exactly equivalent, the RANS limit, obtained when $C_{\varepsilon 2}^{*}=C_{\varepsilon 2}$ for PITM and $\psi=1$ for DES, since, in this case, the equations of the two methods are identical. Therefore, the 
two methods remain equivalent if, starting from the RANS state, the partition of energy is gradually modified by applying successive infinitesimal variations of the coefficients $\psi$ and $C_{\varepsilon 2}^{*}$, respectively, subject to constraint (22). Integrating between the RANS state and some arbitrary state yields

$\int_{C_{\varepsilon 2}}^{C_{\varepsilon 2}^{*}} \frac{1}{x-1} \mathrm{~d} x=-\int_{1}^{\psi} \frac{1}{C_{\varepsilon 2}-y} \mathrm{~d} y$,

and using the definition (9) of $C_{\varepsilon 2}^{*}$, shows that, if

$\psi=1+\left(C_{\varepsilon 2}-C_{\varepsilon 1}\right)(1-r)$,

DES and PITM are H-equivalent.

\subsection{Influence of inhomogeneity}

While the results for locally homogeneous sheared turbulence illustrated the procedure to be followed in formulating an equivalence between methods, the reality is that most flows of engineering relevance are inhomogeneous. Modeled diffusion and transport effects are necessarily reintroduced into Eqs. (13) and (14), which requires some additional restrictions to be applied in the analysis in order to keep a tractable system of equations. It is now assumed that both $k_{\mathrm{M}}$ and $\varepsilon_{\mathrm{M}}$ (rather than only the time-scale $\tau=k_{\mathrm{M}} / \varepsilon_{\mathrm{M}}$ ), are in equilibrium along mean streamlines (for fully developed flows in straight ducts this is exactly satisfied). The system of equations that describe both the PITM and DES methods then reads

$P_{\mathrm{M}}-\psi \varepsilon_{\mathrm{M}}+D_{\mathrm{M}}=0$,

$C_{\varepsilon 1} \frac{\varepsilon_{\mathrm{M}}}{k_{\mathrm{M}}} P_{\mathrm{M}}-C_{\varepsilon 2}^{*} \frac{\varepsilon_{\mathrm{M}}^{2}}{k_{\mathrm{M}}}+D_{\varepsilon}=0$.

\subsubsection{Case of self-consistent methods}

For the PITM $(\psi=1)$, assuming self-consistency $\left(\delta \varepsilon_{\mathrm{M}}=\right.$ 0 ), and following the same procedure as for homogeneous shear, but now including also the infinitesimal variations $\delta D_{\mathrm{M}}$ and $\delta D_{\varepsilon}$ in the system (24)-(25), the resulting equation for the infinitesimal perturbations is

$$
\begin{aligned}
& \delta P_{\mathrm{M}}+\delta D_{\mathrm{M}}=0, \\
& C_{\varepsilon 1} \frac{\varepsilon_{\mathrm{M}}}{k_{\mathrm{M}}} P_{\mathrm{M}}\left(\frac{\delta P_{\mathrm{M}}}{P_{\mathrm{M}}}-\frac{\delta k_{\mathrm{M}}}{k_{\mathrm{M}}}\right)- \\
& C_{\varepsilon 2}^{*} \frac{\varepsilon_{\mathrm{M}}^{2}}{k_{\mathrm{M}}}\left(\frac{\delta C_{\varepsilon 2}^{*}}{C_{\varepsilon 2}^{*}}-\frac{\delta k_{\mathrm{M}}}{k_{\mathrm{M}}}\right)+\delta D_{\varepsilon}=0 .
\end{aligned}
$$

Turbulent diffusion terms $D_{\mathrm{M}}$ and $D_{\varepsilon}$ are usually modeled as

$D_{\mathrm{M}}=\frac{\partial}{\partial x_{k}}\left(C \frac{k_{\mathrm{M}}^{2}}{\varepsilon_{\mathrm{M}}} \frac{\partial k_{\mathrm{M}}}{\partial x_{k}}\right)$

and

$D_{\varepsilon}=\frac{\partial}{\partial x_{k}}\left(\frac{C}{\sigma_{\varepsilon}} \frac{k_{\mathrm{M}}^{2}}{\varepsilon_{\mathrm{M}}} \frac{\partial \varepsilon_{\mathrm{M}}}{\partial x_{k}}\right)$.
The perturbation of the modeled energy $\delta k_{\mathrm{M}}$ is introduced in these two relations. As mentioned at the beginning of section 3.2, the objective of the analysis is to investigate how to enforce a variation $\delta k_{\mathrm{M}}$ representing a given infinitesimal percentage of the modeled energy $k_{\mathrm{M}}$, such that the relative variation $\delta k_{\mathrm{M}} / k_{\mathrm{M}}$ is imposed and constant throughout the domain. Therefore, writing the perturbed energy $k_{\mathrm{M}}+\delta k_{\mathrm{M}}$ as $k_{\mathrm{M}}\left(1+\delta k_{\mathrm{M}} / k_{\mathrm{M}}\right)$, the factor in parenthesis is independent of spatial coordinates, such that it can be easily shown that Eqs. (28) and (29) yield

$\frac{\delta D_{\mathrm{M}}}{D_{\mathrm{M}}}=3 \frac{\delta k_{\mathrm{M}}}{k_{\mathrm{M}}}$,

$\frac{\delta D_{\varepsilon}}{D_{\varepsilon}}=2 \frac{\delta k_{\mathrm{M}}}{k_{\mathrm{M}}}$

Thus, using the 6 equations (24)-(25)-(26)-(27)-(30)-(31) and in the 6 unknowns, $\delta C_{\varepsilon 2}^{*}, \delta P_{\mathrm{M}}, \delta D_{\mathrm{M}}, \delta D_{\varepsilon}, D_{\mathrm{M}}$ and $D_{\varepsilon}$, the following relation is obtained

$\delta C_{\varepsilon 2}^{*}=3\left(\frac{C_{\varepsilon 2}^{*}}{C_{\varepsilon 1}}-1\right) C_{\varepsilon 1} \frac{\delta k_{\mathrm{M}}}{k_{\mathrm{M}}}$.

The same procedure can be followed for the DES system,

$P_{\mathrm{M}}-\psi \varepsilon_{\mathrm{M}}+D_{\mathrm{M}}=0$

$C_{\varepsilon 1} \frac{\varepsilon_{\mathrm{M}}}{k_{\mathrm{M}}} P_{\mathrm{M}}-C_{\varepsilon 2} \frac{\varepsilon_{\mathrm{M}}^{2}}{k_{\mathrm{M}}}+D_{\varepsilon}=0$,

by introducing the infinitesimal modification $\delta \psi$, leading to

$\delta \psi=-3\left(\frac{C_{\varepsilon 2}}{C_{\varepsilon 1}}-\psi\right) \frac{\delta k_{\mathrm{M}}}{k_{\mathrm{M}}}$.

Similar to the equilibrium layer case, using (32) and (35), and integrating between the RANS state $\left(C_{\varepsilon 2}^{*}=C_{\varepsilon 2}\right.$ and $\left.\psi=1\right)$ and an arbitrary state, it is seen that PITM and DES remain H-equivalent if

$\psi=1+\frac{1}{C_{\varepsilon 1}}\left(C_{\varepsilon 2}-C_{\varepsilon 1}\right)(1-r)$

Comparing this relation with (23), it is observed that the only difference is the slope of the linear function, that differs by a factor $C_{\varepsilon 1}$. This same type of influence of the diffusion term was also found by Rumsey et al. [27] in their nullcline analysis of two-equation models. Note that, in the analysis leading to (36), the self-consistency of the hybrid approaches has been assumed ( $\varepsilon_{\mathrm{M}}$ constant), but the postulate of Section 2 has not been invoked. It is worth mentioning that the opposite choice ( $\varepsilon_{\mathrm{M}}$ not constant) can be made, at the price of restricting the analysis to eddy-viscosity models, as shown in the next section. 


\subsubsection{Accounting for departure from self-consistency}

Although self-consistency is a sensible assumption for approaches based on the application of a filter, as introduced in section 2.1, the complexity of the models and the unavoidable simplifications used in their derivation can be at the origin of small variations of the dissipation rate with the cutoff wavenumber or frequency. In the case of DES based on two-equations models or second moment closures, due to the empirical modification of the dissipative term in the subfilter energy equation, the scale $\varepsilon$ does not really represent the physical dissipation rate anymore, except in the RANS limit. Therefore, the issue arises as to whether the analysis above is robust to a possible departure from self-consistency, i.e., robust to variations of the modeled dissipation rate $\varepsilon_{\mathrm{M}}$ with the energy partition. This additional variation makes the above analysis more complex and, eventually, intractable. This difficulty can be circumvented by using an eddy-viscosity hypothesis to evaluate subfilter production

$P_{\mathrm{SFS}}=2 C_{\mu} \frac{k_{\mathrm{sFS}}^{2}}{\varepsilon_{\mathrm{sFs}}} \tilde{S}_{i j} \tilde{S}_{i j}$

The $C_{\mu}$ coefficient can be either constant or function of turbulent invariants, depending on the linear or nonlinear character of the constitutive relation leading to Eq. (37). In order to simplify the analysis, only linear eddy-viscosity models are considered below. The case of nonlinear models is treated in Appendix A.

In the case of linear eddy-viscosity models, the ensemble-averaged subfilter production $P_{\mathrm{M}}=\overline{\left(P_{\mathrm{SFs}}\right)}$ can now be made non-dimensional using $k_{\mathrm{M}}, \varepsilon_{\mathrm{M}}$ and $S$, by introducing the new correlation coefficient

$\gamma^{\prime}=\frac{\overline{C_{\mu} \frac{k_{\mathrm{sFs}}^{2}}{\varepsilon_{\mathrm{SFs}}} \tilde{S}_{i j} \tilde{S}_{i j}}}{C_{\mu} \frac{k_{\mathrm{M}}^{2}}{\varepsilon_{\mathrm{M}}} S^{2}}$,

such that

$P_{\mathrm{M}}=2 \gamma^{\prime} C_{\mu} \frac{k_{\mathrm{M}}^{2}}{\varepsilon_{\mathrm{M}}} S^{2}$.

Again, introducing infinitesimal variations of the variables involved in (39), including for the dissipation rate $\varepsilon_{M}$, the relative variation $\delta P_{\mathrm{M}} / P_{\mathrm{M}}$ of the production term is

$\frac{\delta P_{\mathrm{M}}}{P_{\mathrm{M}}}=\frac{\delta \gamma^{\prime}}{\gamma^{\prime}}+2 \frac{\delta S}{S}+2 \frac{\delta k_{\mathrm{M}}}{k_{\mathrm{M}}}-\frac{\delta \varepsilon_{\mathrm{M}}}{\varepsilon_{\mathrm{M}}}$.

Therefore, with the additional variable $\delta \varepsilon_{\mathrm{M}}$ and the additional equation (40), the same analysis as in section 3.3.1 leads to

$\delta C_{\varepsilon 2}^{*}=\left(C_{\varepsilon 2}^{*}-C_{\varepsilon 1}\right) \frac{P_{\mathrm{M}}}{\varepsilon_{\mathrm{M}}}\left[\frac{\delta k_{\mathrm{M}}}{k_{\mathrm{M}}}-\frac{\delta \gamma^{\prime}}{\gamma^{\prime}}-2 \frac{\delta S}{S}\right]$,

for the PITM system and to

$\delta \psi=-\frac{\left(C_{\varepsilon 2}-C_{\varepsilon 1} \psi\right)}{C_{\varepsilon 2}} \frac{P_{\mathrm{M}}}{\varepsilon_{\mathrm{M}}}\left[\frac{\delta k_{\mathrm{M}}}{k_{\mathrm{M}}}-\frac{\delta \gamma^{\prime}}{\gamma^{\prime}}-2 \frac{\delta S}{S}\right]$, for the DES system. Invoking the postulate of equivalence presented in Section 2, i.e., if two methods are initially $\mathrm{H}$ equivalent, the same modification of the low-order statistics of the resolved scales $\delta S$ and $\delta \gamma^{\prime}$ are obtained for the same modification of the modeled energy $\delta k_{\mathrm{M}}$, the two methods remain equivalent if the relation

$\frac{\delta C_{\varepsilon 2}^{*}}{\left(C_{\varepsilon 2}^{*}-C_{\varepsilon 1}\right)}=-\frac{C_{\varepsilon 2} \delta \psi}{\left(C_{\varepsilon 2}-C_{\varepsilon 1} \psi\right)}$,

is satisfied. Again, integrating between the RANS state and an arbitrary state yields the equivalence criterion

$\psi=1+\frac{1}{C_{\varepsilon 1}}\left(C_{\varepsilon 2}-C_{\varepsilon 1}\right)\left(1-r^{C_{\varepsilon 1} / C_{\varepsilon 2}}\right)$.

It is worth pointing out that Eq. (44) is very close to Eq. (36), since the power $C_{\varepsilon 1} / C_{\varepsilon 2}$ is about 0.75 , and $r$ only varies between 0 and 1 . As will be illustrated in section 3.4 by translating the relations in terms of length scales, these equivalence criteria are very close to each other.

\subsection{Link between energy partitioning, turbulent scales and grid step}

Consequences of the equivalence criteria obtained in the last section can be further explored. In the system of equations of two-equation or second-moment-closure-based DES, in the regions treated in LES, the $\psi$ coefficient is set to

$\psi=\frac{k_{\mathrm{M}}^{3 / 2}}{\varepsilon_{\mathrm{M}} L}$,

that is, the dissipation term in the $k_{\mathrm{M}}$ equation is $k_{\mathrm{M}}^{3 / 2} / L$, where the length scale $L=C_{\mathrm{DES}} \Delta$ is directly proportional to the local grid step. Using the self-consistency hypothesis $\left(\varepsilon=\varepsilon_{\mathrm{M}}\right)$, Eq. (45) shows that the length scale to be used in DES to ensure H-equivalence with PITM is

$L=\frac{r^{3 / 2}}{\psi(r)} L_{\text {int }}$,

where

$L_{\text {int }}=\frac{k^{3 / 2}}{\varepsilon}$

is the integral length scale. Similar to PITM, the fundamental parameter in this relation is the energy ratio

$r=\frac{k_{\mathrm{M}}}{k_{\mathrm{R}}+k_{\mathrm{M}}}=\frac{k_{\mathrm{M}}}{k}$.

As was shown by Schiestel and Dejoan [20] for the PITM and by Fadai-Ghotbi et al. [10] for the T-PITM, an evaluation of $r$ as a function of $\Delta$ can be obtained by integrating the wavenumber spectrum or the Eulerian frequency spectrum, respectively, under Kolmogorov assumptions,

$r=\frac{1}{\beta_{0} \pi^{2 / 3}}\left(\frac{\Delta}{L_{\text {int }}}\right)^{\frac{2}{3}}$. 
Introducing this relation into the length scales $L=k_{\mathrm{M}}^{3 / 2} /(\varepsilon \psi)$ obtained for equilibrium layers or spatially developed flows in section 3 shows that, for the DES method to be equivalent to PITM, the constant coefficient $C_{\mathrm{DEs}}$ must be replaced by the function

$f_{\mathrm{DES}}=\frac{r^{3 / 2} L_{\mathrm{int}}}{\psi \Delta}=\frac{1}{\beta_{0}^{3 / 2} \pi \psi(r)}$.

If the coefficients $C_{\mathrm{DEs}}$ and $\beta_{0}$ are calibrated in such a way that the length scales $L$ obtained from the different equivalence criteria reach the integral scale for the same value of the grid step, figure 2 shows that these criteria lead to very similar variations of the length scale as a function of $\Delta$, and that standard DES is a simple linear approximation of formulation (50), called equivalent-DES.

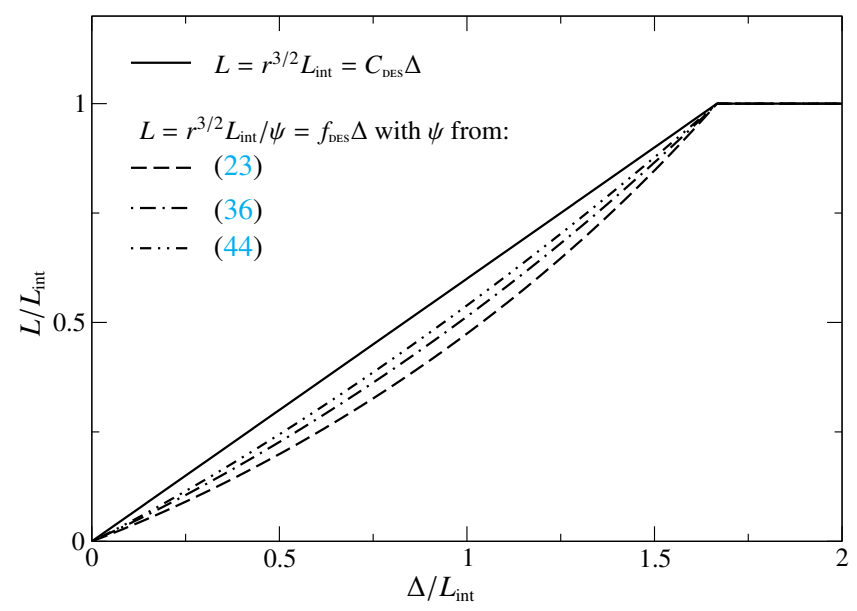

Figure 2: Comparison of the length scales of the original DES and the three versions of equivalent-DES.

\section{Computational validation}

As introduced in section 2.3, the H-equivalence property is concerned with the link between the ensemble-averaged subfilter energy and the variable coefficients $C_{\varepsilon 2}^{*}$ or $\psi$ used to hybridize RANS and LES. Therefore, the analysis does not provide information about the time-dependent solutions of the filtered equations given below (Eq. 51), but only to their low-order statistical properties, in particular the partition of energy. Moreover, the analysis of section 3 provides analytical equivalence criteria only in particular situations of equilibrium turbulence. The purpose of the present computational validation is then twofold

(i) In order to investigate the validity of applying to time-dependent hybrid RANS/LES computations the equivalence criteria obtained by the analysis at the ensemble-averaged level, a channel flow [28] at $R e_{\tau}=$ 395 is computed. In other words, the objective is to examine the statistical properties of a real, timedependent computation using the T-PITM and the
equivalent-DES, whose coefficients satisfy an equivalence criterion. In order to compare the influence of the particular form of the equivalence relation obtained under different assumptions, four different computations are performed using equivalent-DES, using the four length scale relations plotted in figure 2.

(ii) With the purpose of validating the applicability of the equivalence criteria in a case that does not comply with the equilibrium hypothesis of the theoretical analysis, a flow over a periodic hill at bulk Reynolds number $R e_{b}=10595$ is computed [29].

The T-PITM model used for the computations is a second moment closure of the temporally-filtered, incompressible Navier-Stokes equations

$\frac{\partial \tilde{u}_{i}}{\partial t}+\tilde{u}_{k} \frac{\partial \tilde{u}_{i}}{\partial x_{k}}=-\frac{1}{\rho} \frac{\partial \tilde{p}}{\partial x_{i}}+v \frac{\partial^{2} \tilde{u}_{i}}{\partial x_{j} \partial x_{j}}-\frac{\partial \tau_{i j_{\mathrm{SFS}}}}{\partial x_{j}}$,

where $\tilde{u}_{i}$ and $\tilde{p}$ denote the temporally-filtered velocity and pressure, respectively, which are dependent on $\mathbf{x}$ and $t$. The transport equation for the subfilter stress

$\frac{\partial \tau_{i j_{\mathrm{SFS}}}}{\partial t}+\tilde{U}_{k} \frac{\partial \tau_{i j_{\mathrm{SFs}}}}{\partial x_{k}}=P_{i j_{\mathrm{SFs}}}-\varepsilon_{i j_{\mathrm{SFS}}}+\phi_{i j_{\mathrm{SFS}}}^{*}+D_{i j_{\mathrm{SFs}}}^{T}+D_{i j_{\mathrm{SFs}}}^{v}$

is modeled using an adaptation of the Elliptic-Blending Reynolds-Stress model $[30,31]$ to the hybrid temporal LES framework [10]. In this equation, similar to the case of the Reynolds-stress transport equation used in RANS, $P_{i j_{\mathrm{SFS}}}$, $\varepsilon_{i j_{\mathrm{SFS}}}, \phi_{i j_{\mathrm{SFs}}}^{*}, D_{i j_{\mathrm{SFS}}}^{T}$ and $D_{i j_{\mathrm{SFS}}}^{v}$ denote subfilter production, dissipation, velocity-pressure gradient correlation, turbulent diffusion and viscous diffusion tensors, respectively. The main characteristic of this second moment closure is the blending of near-wall and homogeneous forms of the velocitypressure gradient correlation and dissipation tensors, in order to reproduce the wall-blockage effects and the associated two-component limit of turbulence, under the form

$\phi_{i j_{\mathrm{SFS}}}=\left(1-\alpha^{3}\right) \phi_{i j}^{w}+\alpha^{3} \phi_{i j}^{h}$,

$\varepsilon_{i j_{\mathrm{SFS}}}=\left(1-\alpha^{3}\right) \frac{\tau_{i j_{\mathrm{SFS}}}}{k_{\mathrm{SFS}}} \varepsilon_{\mathrm{SFS}}+\alpha^{3} \frac{2}{3} \varepsilon_{\mathrm{sFS}} \delta_{i j}$

where $\alpha$ is a blending parameter, solution of an elliptic relaxation equation

$\alpha-L_{\mathrm{sFS}}^{2} \nabla^{2} \alpha=1$

(for details, see Fadai-Ghotbi et al. [10]). The dissipation rate is obtained via the transport equation

$$
\begin{aligned}
\frac{\partial \varepsilon_{\mathrm{SFS}}}{\partial t}+\tilde{U}_{k} \frac{\partial \varepsilon_{\mathrm{SFS}}}{\partial x_{k}}= & \frac{C_{\varepsilon_{1}}^{\prime} P_{\mathrm{SFs}}-C_{\varepsilon_{2}}^{*} \varepsilon_{\mathrm{SFS}}}{T}+ \\
& \frac{\partial}{\partial x_{l}}\left(\frac{C_{\mu}}{\sigma_{\varepsilon}} \tau_{l m_{\mathrm{SFS}}} T \frac{\partial \varepsilon_{\mathrm{SFs}}}{\partial x_{m}}\right)+v \frac{\partial^{2} \varepsilon_{\mathrm{SFs}}}{\partial x_{k} \partial x_{k}}
\end{aligned}
$$

in which, in order to sensitize the model to the filter width and thus migrate from a RANS to a LES behavior, the $C_{\varepsilon_{2}}^{*}$ coefficient is made a function of the energy ratio $r$, using 

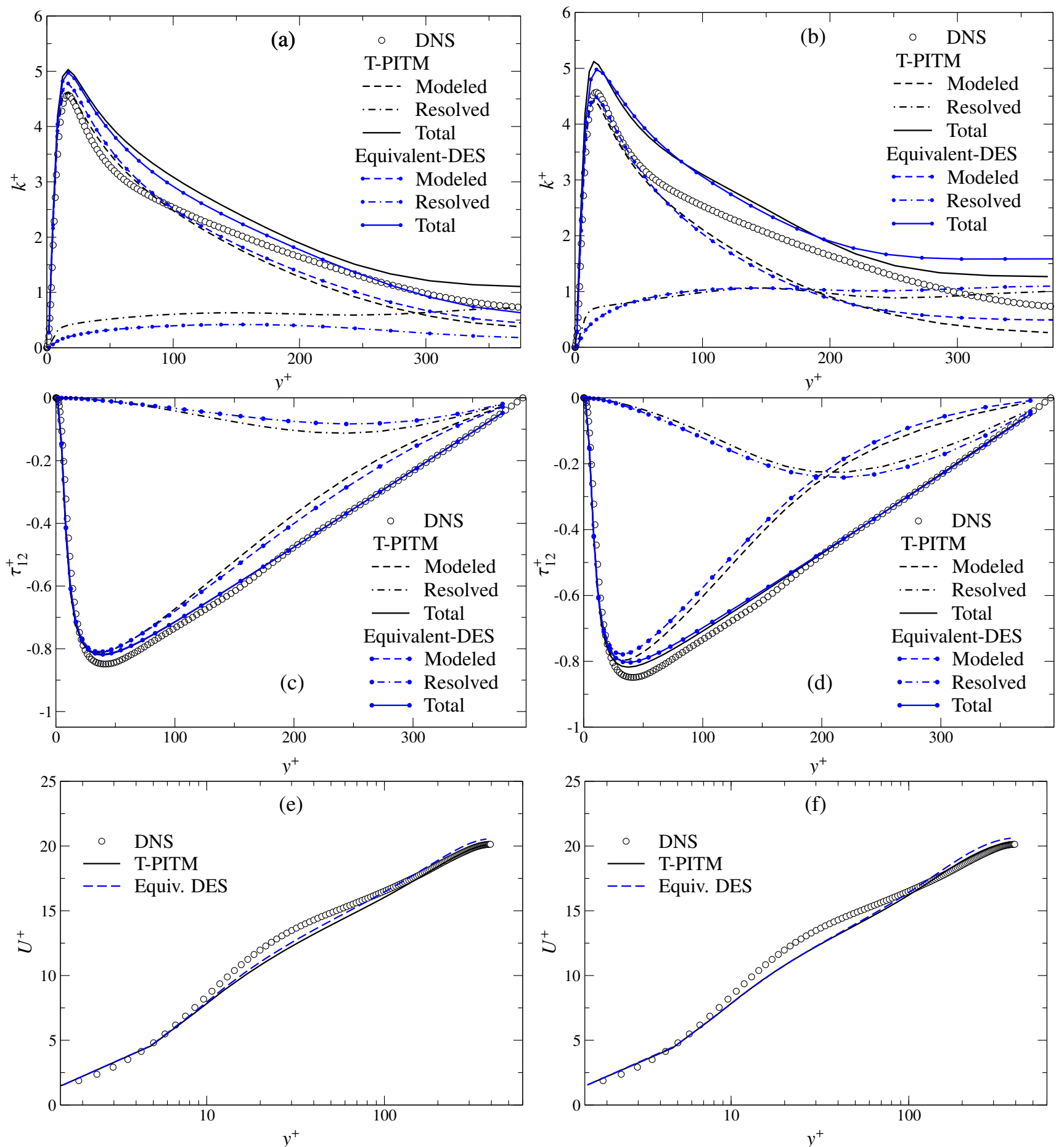

Figure 3: Channel flow: (a,b) Turbulent energy; (c,d) Shear stress; (e,f) Mean velocity profiles. Comparison of the equivalent-DES using relation (44) with the T-PITM. Left: coarse mesh; Right: fine mesh.

Eq. (9). Since $r$ is the ratio of modeled energy $k_{\mathrm{M}}$ to total energy $k$, it can be computed during the simulation, leading to a self-adaptive method, i.e., a method that does not explicitly depend on the grid step, as for instance the scale-adaptive simulation [22], the "universal model" of Perot \& Gadebusch [32], or the dynamic hybrid RANS/LES [33]. Another pos- sibility, which is applied here, is to evaluate $r$ using (49), obtained by analytical integration of a generic spectrum [10].

For the equivalent-DES method, the same transport equations (52) for the subfilter stresses are solved, using the same model (53)-(54)-(55). The only differences with T-PITM are that the $C_{\varepsilon 2}^{*}$ coefficient in the dissipation equation (56) 

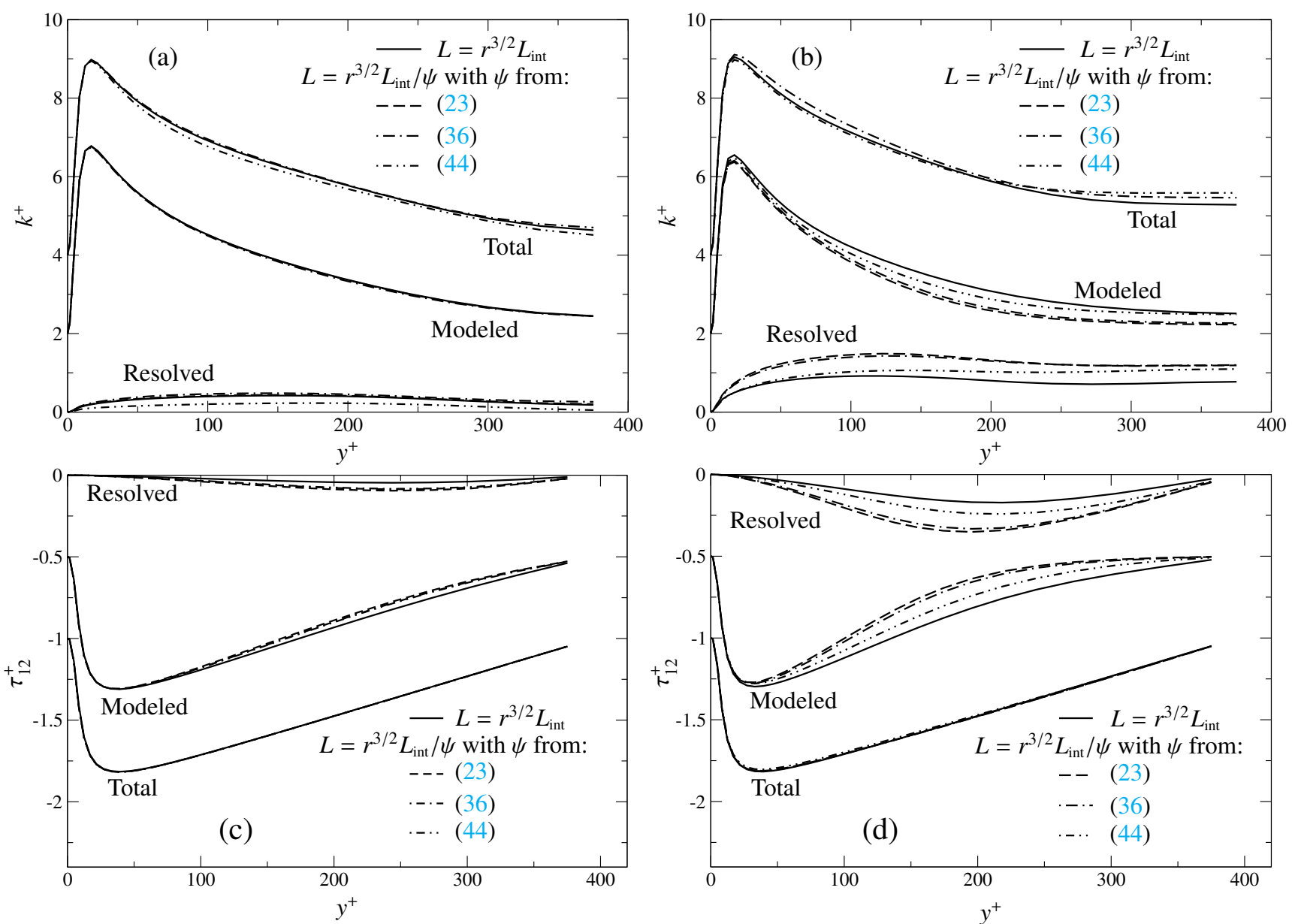

Figure 4: Channel flow: (a,b) Turbulent energy; (c,d) Shear stress. Comparison of the versions of the equivalent-DES obtained using the different length scales shown in figure 2. Left: coarse mesh; Right: fine mesh. Profiles are shifted for clarity.

is constant, and equal to the usual RANS value $C_{\varepsilon 2}$, and the dissipation rate $\varepsilon_{\mathrm{sFs}}$ in Eq. (54) is replaced by

$\max \left(\varepsilon_{\mathrm{SFs}} ; \frac{k_{\mathrm{sFs}}^{3 / 2}}{L}\right)$.

where $L$ is given by Eq. (46). For comparison, the four versions plotted in figure 2 are applied, corresponding to the different forms of the equivalent criterion (23), (36) and (44), and to the linear approximation $L=r^{3 / 2} L_{\mathrm{int}}$.

\subsection{Channel flow}

Computations are performed with Code_Saturne, a second order accurate, parallel, finite volume solver on unstructured grids, developed at EDF [34]. The size of the computational domain is $8 h \times 2 h \times 4 h$ in the streamwise, normalwise and spanwise directions, respectively, where $h$ is the channel half-width, which corresponds to $3160 \times 790 \times 1580$ in wall units. The reference grid is made of about 221,000 cells $(64 \times 54 \times 64)$. The grid resolution in the streamwise and spanwise directions thus corresponds to $\Delta x^{+} \simeq 50$ and $\Delta z^{+} \simeq 25$, respectively. In the wall-normal direction, the grid is clustered in the vicinity of the walls, with a first discretization point located at $y^{+} \simeq 1.5$, and cells of size $\Delta y^{+} \simeq 40$ at the centerline. In order to investigate the evolution with the grid of the solutions, and, in particular, the partition of energy among resolved and modeled scales, a coarser mesh in also used, similar to the reference (fine) grid, but reduced to 55,000 cells.

It can be seen in figure 3 that the predictions of the equivalent-DES method are very close to those of the TPITM. A refinement of the grid leads to a significant modification of the partition of energy and shear stress among resolved and modeled scales, leaving the total energy almost constant, as already observed by Chaouat and Schiestel [5] and Fadai-Ghotbi et al. [7]. Here, the version with relation (44) is used. As will be shown below, the different relations obtained in section 3 yield slightly different results. The model based on (44) is the closest formulation to the T-PITM.

For the two grids, the energy partition given by the two methods is nearly the same, with the same transition from RANS to LES when moving away from the wall. This obser- 
vation is made for the decomposition of $k$ into modeled and resolved parts: the turbulent energy $k$ is dominated by the modeled contribution in the near-wall region, which shows that the model works in RANS mode; far away from the wall, the relative contribution of the resolved part increases, as the model gradually switches to the LES mode. The RANS to LES transition is driven by the ratio of the grid step to the integral length scale that enters the energy ratio $r$ in Eq. (49). Although $r$ is involved in the $C_{\varepsilon 2}^{*}$ coefficient for the T-PITM and the $\psi$ coefficient for the equivalent-DES, the obtained energy partition and the transition from RANS to LES are very similar for the two grids, which validates the equivalence criterion of section 3: although this criterion is established based on the equations for the ensemble-averaged filtered variables, the present results show that two H-equivalent approaches, i.e., whose coefficients satisfy the equivalence criterion, give very similar statistics. The similarity is even more striking for the partition of the shear stress $\tau_{12}$. This result validates the postulate put forth in section 2.3 , and is mainly due to the use of the same closure for the subfilter stress: as soon as the partition of energy among resolved and modeled scales is the same, the length and time scales entering the subfilter stress closure for the two methods are the same, such that the system of equations are identical. However, the mean velocity shown in figure 3 is slightly overpredicted by the equivalent-DES at the center of the channel compared to the T-PITM, but this problem could be easily fixed by a recalibration: the same coefficients as for T-PITM have been used for the equivalent-DES, in order to evaluate the validity of the $\mathrm{H}$-equivalence between the two methods. In particular, the optimal coefficient $\beta_{0}$ entering the evaluation of $r$ should be slightly different. The velocity profile does not reproduce the DNS very well, but it is worth mentioning that the hybrid methods are used in a challenging situation, the RANS to LES transition being activated at the beginning of the log layer. The results could be improved by protecting the log layer from the transition to the LES mode, either by coarsening the grid and by introducing a shield function, as done for instance in DDES [35], but such a method would be in contradiction with the purpose of showing the equivalence between the two approaches, within the theoretical framework of this paper.

Figure 4 shows, for the two grids, a comparison between the versions of the equivalent-DES obtained using the four length scales plotted in figure 2. It can be seen that the formulations obtained using different hypotheses lead to a slight variability of the partition among resolved and modeled scales, without much affecting the total. This variability is reduced when the grid is coarsened, because the hybrid methods gradually approach the RANS limit, for which they are exactly equivalent. The formulation using relation (44) is used in the comparison with the T-PITM in figure 3 and for the periodic hill case in section 4.2 , because it provides the closest results to the T-PITM. It is interesting to remark that this formulation is obtained based on the less restrictive hypotheses: the influence of diffusion is accounted for, as well as the departure from self-consistency. The fact that it is necessary to use the eddy-viscosity assumption (37) for the subfilter production in order to obtain the analytical form of the equivalence criterion is not detrimental to the validity of this relation.
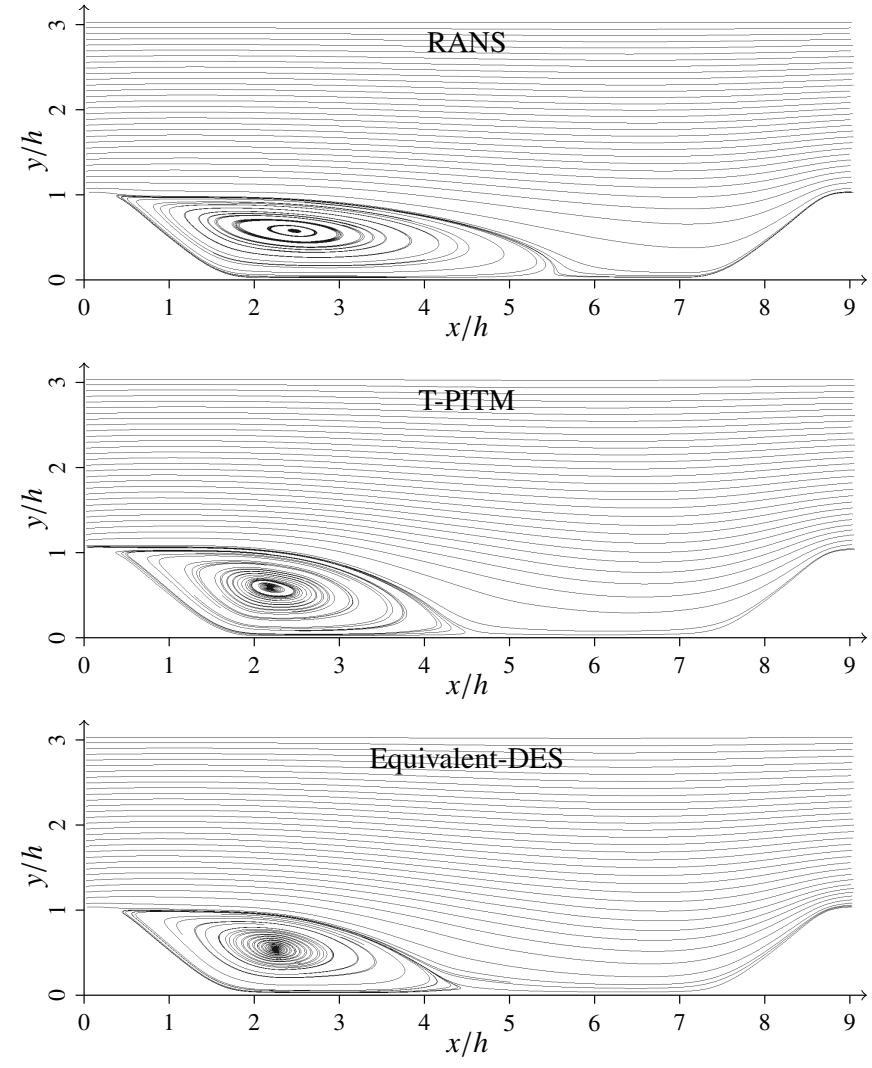

Figure 5: Periodic hill flow. Streamlines obtained using the fine grid.

\subsection{Periodic hill flow}

The validity of the equivalence criterion is now assessed in a massively separated flow, far from the equilibrium assumptions used in the theoretical derivation, the periodic hill flow at $R e_{b}=10595$ [29], for which PITM proved successful [36]. The size of the computational domain is $9 h \times 3.035 h \times 4.5 h$ ( $h$ is henceforth the height of the hill). The reference grid contains 960,000 cells $(160 \times 100 \times 60)$. The evolution with the grid of the solution and, in particular, the partition among resolved and modeled scales, is investigated using a coarser grid, reduced to 240,000 cells.

Although the purpose here is not to compare results given by the hybrid and RANS methods, streamlines are plotted in figure 5 in order to provide the reader with a global overview of the flow fields under consideration. It clearly appears that the RANS model gives too long a recirculation region, with a reattachment point located at $x / h=5.3$, to be compared with $x / h=4.7$ for the reference fine LES [29]. The two 

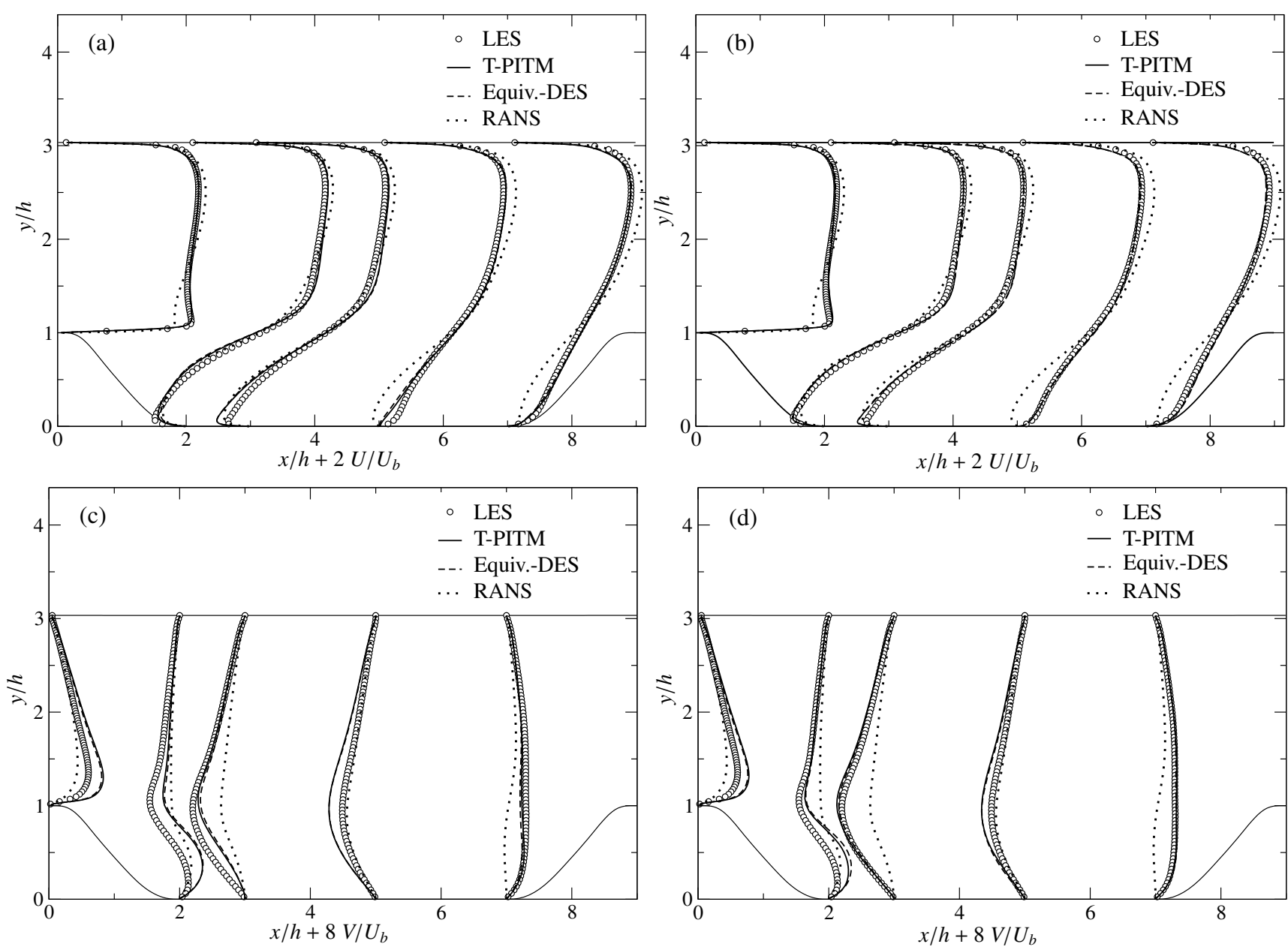

Figure 6: Periodic hill flow: (a,b) streamwise velocity; (c,d) normalwise velocity. Left: coarse mesh; Right: fine mesh.

hybrid RANS/LES methods, T-PITM and equivalent-DES, yield very close reattachment points, both at $x / h=4.5$. Note that on the coarse grid (streamlines not shown here), this point is moved to $x / h=5.0$ for the two hybrid methods.

Profiles extracted at $x / h=0,2,3,5$ and 7 , respectively, are shown in figures 6 to 8 . Despite the fact that such a flow strays from the theoretical framework of Sec. 3, it can be seen that the predictions of the equivalent-DES method is in overall strong agreement with T-PITM, for the two grids, even in the recirculation region (see profiles at $x / h=2,3$ and 5). Figure 6 shows mean flow quantities: profiles of the streamwise and normalwise velocities. It is observed that, although the mean velocity fields given by the hybrid methods are dependent on the grid refinement, they remain in close agreement with each other in the domain. It also appears that, when the grid is refined, the hybrid solutions approach the LES solution, thus exhibiting the expected behavior, i.e., a migration from a RANS-type to a LES-type solution.

Besides the H-equivalence between T-PITM and equivalent-DES, it is worth emphasizing that the agreement with the reference LES is satisfactory. For such out-ofequilibrium flows, seamless hybrid RANS/LES methods significantly improve the predictions of their parent RANS model, independently of the hybridization method. In particular, in the recirculation region, which is far from equilibrium, RANS is far from fine LES, and the hybrid RANS/LES models gradually depart from RANS and tend to LES with grid refinement. In contrast, in regions close to equilibrium, such as the upper half of the channel, RANS is close to LES, such that the room for improvement is not sufficient for hybrid RANS/LES methods to exhibit a major superiority. Figure 7 shows the profiles of the Reynolds stresses for the two grids. It is clearly observed that RANS yields correct profiles for all the components in the upper half of the channel, but is not providing the correct levels of fluctuations in nonequilibrium regions, such as the recirculation bubble, and, above all, in the separated shear layer. The lack of turbulent stresses is at the origin of the above-mentioned overestimation of the recirculation length. Hybrid RANS/LES methods are able to compensate for this under-prediction by resolv- 
14

Ch. Tries et al. / Accepted for publication in Computers $\mathcal{E}$ Fluids
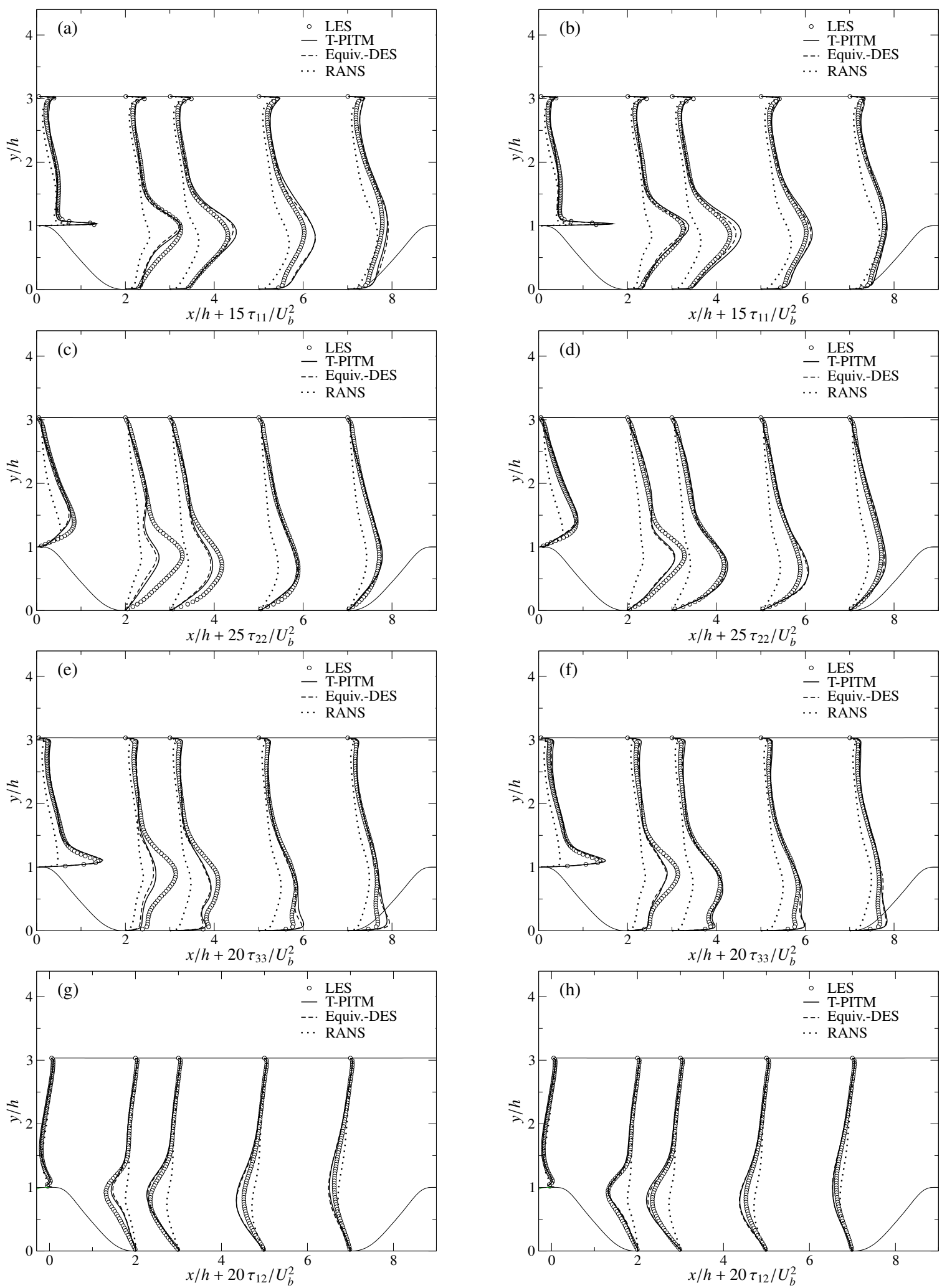

Figure 7: Periodic hill flow: Total Reynolds stresses. (abb) $\tau_{11}$; (cd) $\tau_{22}$; (eff) $\tau_{33}$; (g,h) $\tau_{12}$. Left: coarse mesh; Right: fine mesh. 

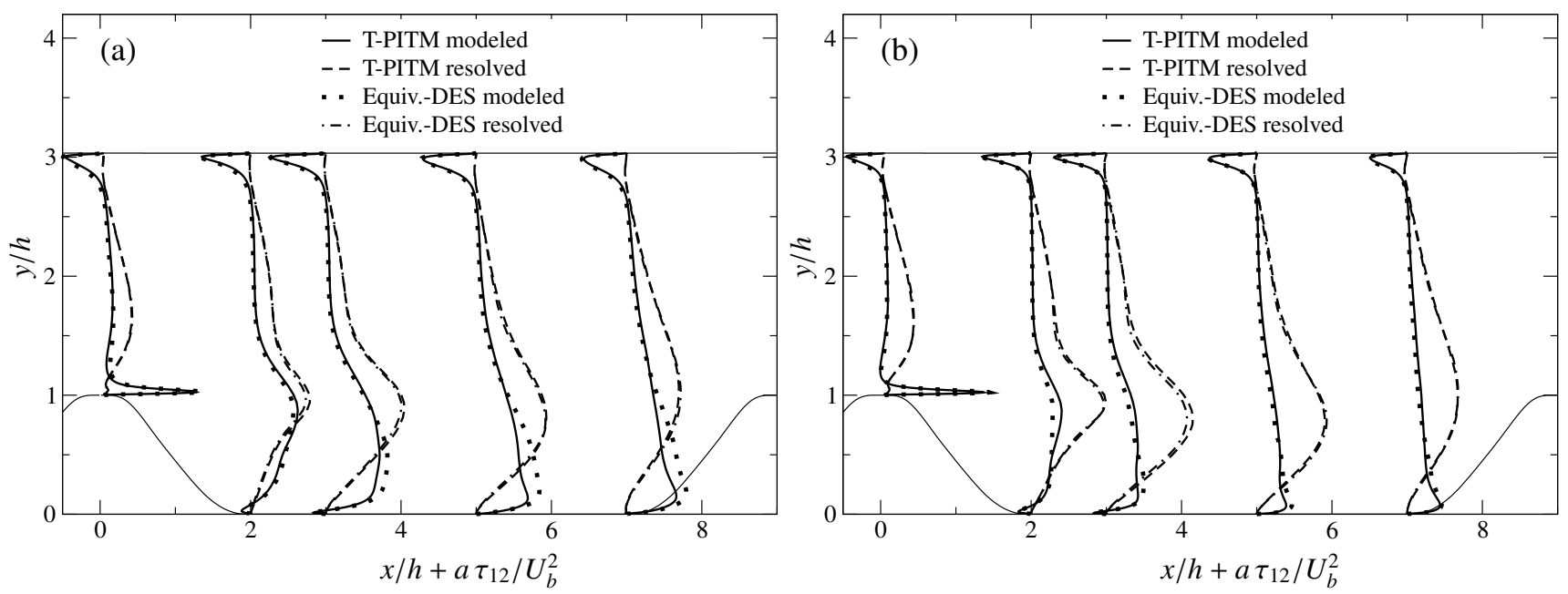

Figure 8: Periodic hill flow. Decomposition of the shear stress $\tau_{12}$ into modeled and resolved parts for the T-PITM and Equivalent-DES methods. (a) Coarse mesh; (b) Fine mesh. For the sake of clarity, a different factor $a$ is applied to modeled $(a=-300)$ and resolved $(a=-30)$ parts.

ing a significant part of the stresses. Indeed, figure 8 shows that, in the separated shear layer, the main contribution to the shear stress is due to the resolved motion, and that the weight of the resolved part increases with grid refinement. It is also noticeable that the Reynolds stresses predicted by the two hybrid RANS/LES methods are virtually identical in the whole domain and for the two grids. Again, the hybrid RANS/LES solutions gradually depart from RANS and approach LES when the grid is refined.

With the objective of validating the analytical results of section 3 , figure 8 is particularly useful. Indeed, for the two grids, the relative contributions of the resolved and modeled motions to the total shear stress are very similar for the two hybrid methods. The partition is significantly modified with grid refinement, but in the same way for the two methods, as expected from the analysis of section 3. These results, obtained in a case outside of the range of application of the theoretical analysis, suggest that different hybrid methodologies can be made H-equivalent with a relevant choice of the parameters controlling the transition from RANS to LES. Therefore, the subfilter scale closure and the hybridization method can be chosen independently of each other.

\section{Conclusion}

The analysis and the results shown in the present article lead to the conclusion that, under some conditions, an equivalence relation, called $H$-equivalence, can be defined between two different seamless hybrid RANS/LES methods, ensuring that the two methods yield the same partition of energy among resolved and unresolved scales. The obtained equivalence criteria lead to constraints to be satisfied by the variable coefficients introduced to enforce the migration from a RANS to a LES behavior. The $\mathrm{H}$-equivalence is estab- lished without any assumption about the closure of the subfilter stresses, provided that the methods are self-consistent, i.e., the modeled dissipation rate is independent on the cutoff wavenumber, as expected from a filtered approach. If selfconsistency is not exactly satisfied, H-equivalence appears to be established only for eddy-viscosity models. Moreover, the equivalence criteria were empirically assessed for the flow upon a periodic hill, involving massive separation and turbulence far from equilibrium, thus excluded from the theoretical framework of the present article. The encouraging agreement between the two hybrid methods observed for this case suggests that the equivalence criteria are valid beyond the restrictive framework of equilibrium flows. This result corroborates the fact that Kubacki and Dick [11] observed a similar equivalent behavior between hybrid methods, when simulating plane impinging jets.

The length scale appearing in DES to control the partition of energy is, in the version H-equivalent to PITM, parametrized by the energy ratio $r$ (modeled energy over total energy), and does not explicitly depend on the grid step. Nonetheless, if $r$ is evaluated from a Kolmogorov spectrum assumption, as usual in PITM, it can be shown that the standard DES length scale $L=C_{\mathrm{DES}} \Delta$ is recovered, however with a variable $C_{\mathrm{DES}}$ coefficient.

Now, as shown by Fadai-Ghotbi et al. [10], the spatialfiltering formalism used for PITM in homogeneous flows can be replaced by temporal filtering in the case of inhomogeneous flows, leading to T-PITM. Therefore, in most of the practical applications, that are inhomogeneous and stationary, the H-equivalence criterion applied to DES and TPITM suggests that DES can be interpreted as a model for the subfilter stress appearing in the temporally filtered NavierStokes equations, compatible with the limit of infinite temporal filter width (RANS) in stationary flows. Therefore, the 


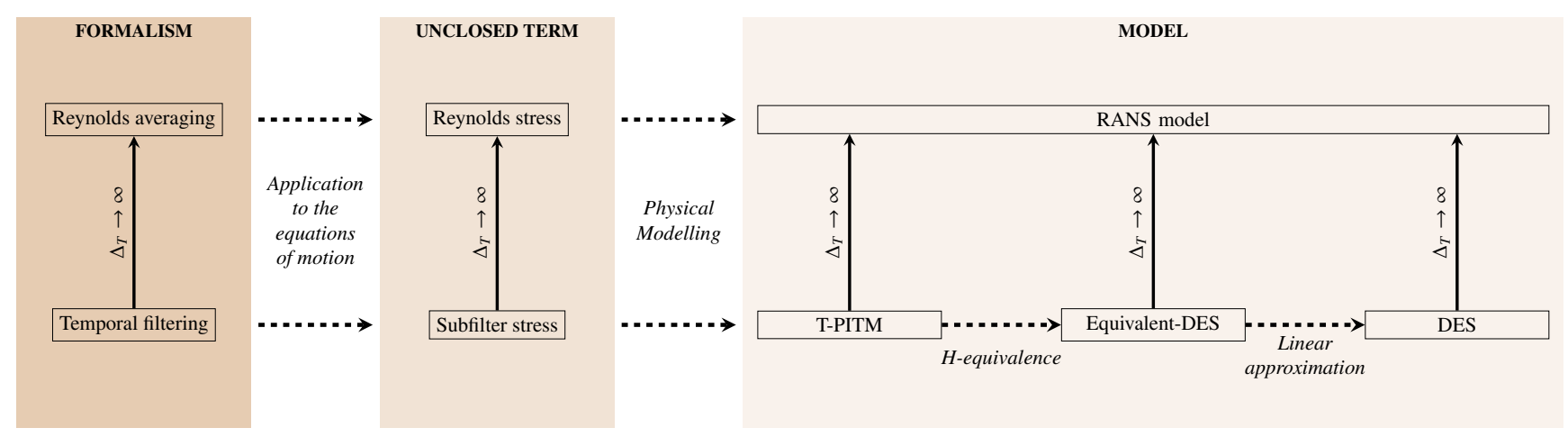

Figure 9: Schematic view of the mathematical and modeling process leading to hybrid RANS/LES methods (here, TPITM, equivalent-DES and DES), in the case of inhomogeneous, stationary flows.

$\mathrm{H}$-equivalence criterion can be used to provide a formalism to link DES to the equations of motion. Indeed, as illustrated in figure 9, DES can be derived by a four step modeling process: the formalism of temporal filtering is first introduced in the equations of motion, leading to unclosed equations; second, a physical model (i.e., a model based on the physical interpretation of the terms) is introduced for the subfilter stress, such as the T-PITM, based on a spectral partitioning of the turbulent field; third, the H-equivalence criterion is applied, as shown in section 3 , in order to obtain a hybrid method (denoted as equivalent-DES) that gives loworder statistical results similar to the T-PITM, with a control of the energy partition via the subfilter energy equation rather than the dissipation equation; fourth, the length scale entering this approach is approximated by a linear length scale, leading to standard DES. It is worth emphasizing that during all this process, the compatibility of the equations with the Reynolds-averaged equations and the corresponding RANS model is preserved in the limit of infinite filter widths. Therefore, the variables entering the DES approach can be interpreted as temporally-filtered variables, which facilitates the understanding and modeling of the physical role of the terms of the different transport equations and the comparison of the results with existing databases. This interpretation is a possible answer to the issue raised by Spalart [9] of the missing link between the DES flow field and the exact or DNS flow field.

\section{Acknowledgments}

This work was granted access to the HPC resources of IDRIS under the allocation 2010-020912 made by GENCI (Grand Equipement National de Calcul Intensif) and to the computing facilities of the MCIA (Mésocentre de Calcul Intensif Aquitain) of the University of Bordeaux and of the University of Pau.

\section{Appendix A. Extension of the analysis of section 3.3.2 to nonlinear eddy-viscosity models}

For the sake of generality, $C_{\mu}$ is now assumed to be variable, under a form typical to many nonlinear eddy-viscosity models and explicit algebraic stress models,

$$
C_{\mu}=\frac{a_{0}}{1+a_{1} \tilde{S}_{i j} \tilde{S}_{i j} \frac{k_{\mathrm{sFs}}^{2}}{\varepsilon_{\mathrm{sFs}}^{2}}},
$$

where $a_{0}$ and $a_{1}$ are constants. The averaged subfilter production can now be made non-dimensional using $k_{\mathrm{M}}, \varepsilon_{\mathrm{M}}, S$ and $C_{\mu_{\mathrm{M}}}=a_{0} /\left(1+a_{1} S^{2} k_{\mathrm{M}}^{2} / \varepsilon_{\mathrm{M}}\right)$

$$
\gamma^{\prime}=\frac{P_{\mathrm{M}}}{2 C_{\mu_{\mathrm{M}}} \frac{k_{\mathrm{M}}^{2}}{\varepsilon_{\mathrm{M}}} S^{2}},
$$

thus redefining $\gamma^{\prime}$. Since $\delta C_{\mu_{\mathrm{M}}}$ can be written as

$\frac{\delta C_{\mu_{\mathrm{M}}}}{C_{\mu_{\mathrm{M}}}}=-2 \beta\left(\frac{\delta k_{\mathrm{M}}}{k_{\mathrm{M}}}+\frac{\delta S}{S}-\frac{\delta \varepsilon_{\mathrm{M}}}{\varepsilon_{\mathrm{M}}}\right)$,

where $\beta=a_{1} \eta^{2} /\left(1+a_{1} \eta^{2}\right)$, and $\eta=S k_{\mathrm{M}} / \varepsilon_{\mathrm{M}}$, the variation $\delta P_{\mathrm{M}}$ is now

$\frac{\delta P_{\mathrm{M}}}{P_{\mathrm{M}}}=\frac{\delta \gamma^{\prime}}{\gamma^{\prime}}+2(1-\beta) \frac{\delta S}{S}+2(1-\beta) \frac{\delta k_{\mathrm{M}}}{k_{\mathrm{M}}}-(1-2 \beta) \frac{\delta \varepsilon_{\mathrm{M}}}{\varepsilon_{\mathrm{M}}}$.(A.4)

This modified equation now leads to

$\delta C_{\varepsilon 2}^{*}=\left(C_{\varepsilon 2}^{*}-C_{\varepsilon 1}\right) P_{\mathrm{M}} \frac{\left[\left(1+a_{1} \eta^{2}\right) \frac{\delta \gamma^{\prime}}{\gamma^{\prime}}-\frac{\delta k_{\mathrm{M}}}{k_{\mathrm{M}}}+2 \frac{\delta S}{S}\right]}{a_{1} \eta^{2}\left(\varepsilon_{\mathrm{M}}-P_{\mathrm{M}}\right)+\varepsilon_{\mathrm{M}}}$,

for the PITM system and to

$\delta \psi=\frac{\left(C_{\varepsilon 1} \psi-C_{\varepsilon 2}\right)}{C_{\varepsilon 2}} P_{\mathrm{M}} \frac{\left[\left(1+a_{1} \eta^{2}\right) \frac{\delta \gamma^{\prime}}{\gamma^{\prime}}-\frac{\delta k_{\mathrm{M}}}{k_{\mathrm{M}}}+2 \frac{\delta S}{S}\right]}{a_{1} \eta^{2}\left(\varepsilon_{\mathrm{M}}-\frac{C_{\varepsilon 1}}{C_{\varepsilon 2}} P_{\mathrm{M}}\right)+\varepsilon_{\mathrm{M}}}$,

for the DES system. The application of the postulate yields

$\beta \frac{\delta C_{\varepsilon 2}^{*}}{\left(C_{\varepsilon 2}^{*}-C_{\varepsilon 1}\right)}=-\frac{C_{\varepsilon 2} \delta \psi}{\left(C_{\varepsilon 2}-C_{\varepsilon 1} \psi\right)}$, 
where

$$
\beta=\frac{1+a_{1} \eta^{2}\left(1-\frac{P_{\mathrm{M}}}{\varepsilon_{\mathrm{M}}}\right)}{1+a_{1} \eta^{2}\left(1-\frac{C_{\varepsilon 1}}{C_{\varepsilon 2}} \frac{P_{\mathrm{M}}}{\varepsilon_{\mathrm{M}}}\right)} .
$$

Equation (A.7) is similar to Eq. (43), except for the presence of the $\beta$ coefficient, and shows that an equivalence criterion can be found for non-linear eddy-viscosity models. However, in contrast to the previous cases, this equation cannot be analytically integrated, because of the variable coefficient $\beta$. It is worth pointing out that, if the variations of $\beta$ are not too strong, which is expected if subfilter turbulence is close to equilibrium $\left(P_{\mathrm{M}} \simeq \varepsilon_{\mathrm{M}}\right)$, the approximate relation

$\psi=1+\frac{1}{C_{\varepsilon 1}}\left(C_{\varepsilon 2}-C_{\varepsilon 1}\right)\left(1-r^{\beta C_{\varepsilon 1} / C_{\varepsilon 2}}\right)$,

is obtained, which just differs from Eq. (44) by the factor $\beta$. If $a_{1}$ is set to zero in Eq. (A.8), the case of linear eddy-viscosity models, Eq. (44), is recovered.

\section{Appendix B. Decaying isotropic turbulence}

One of the particularities of the approaches derived in the present paper is that the key parameter is the energy ratio $r$. As shown by Eq. (49), this ratio can be evaluated as a function of the grid step if a Kolmogorov spectrum is assumed. In this case, the value of the coefficient $\beta_{0}$ is directly related to the Kolmogorov constant $C_{K}$ by $\beta_{0}=2 /\left(3 C_{K}\right) \simeq 0.44$. Therefore, contrary to standard DES, for which the coefficient $C_{\mathrm{DES}}$ is calibrated against decaying isotropic turbulence, the function $f_{\mathrm{DES}}$ of the equivalent DES is entirely determined by the analysis and is not a calibrated value, such that the ability of the $f_{\mathrm{DES}}$ function to provide the correct level of dissipation needs to be validated.

The validation is performed in the case of incompressible decaying isotropic turbulence. The initial velocity field at $R e_{\lambda}=104.5$ and the reference DNS data are from Wray [37]. The triply-periodic domain is a $(2 \pi)^{3}$ box. Computations are performed with Code_Saturne. Two grids, consisting of $32^{3}$ and $64^{3}$ cells, are used. The initial velocity field is obtained by filtering the DNS field using a cutoff filter adapted to the grid step. The initial fields of the subfilter quantities are obtained by running a preliminary computation with frozen velocities (initial velocity field). Figure B.10 shows the decay of the resolved turbulent energy, for the two grids. This test case is particularly relevant since the effect of the variable function $\psi(r)$ can be observed as a function of the grid step. First, it can be seen that, with the theoretical value 0.44 of the coefficient $\beta_{0}$, the two versions of the equivalent DES compared in figure B.10 provide a correct amount of dissipation, very close to the standard DES with the optimized coefficient $C_{\mathrm{DES}}=0.6$. On the coarser mesh, a hierarchy cannot be established among the three versions of DES. On the contrary, on the finer mesh, the equivalent DES with a linear function $\psi(r)$, Eq. (23), which is derived using the homogeneity hypothesis, gives a slightly better time-evolution of the spectra.

\section{References}

[1] J. Fröhlich, D. von Terzi, Hybrid LES/RANS methods for the simulation of turbulent flows, Prog. Aerosp. Sci. 44 (5) (2008) 349-377.

[2] P. Sagaut, S. Deck, M. Terracol, Multiscale and multiresolution approaches in turbulence, Imperial College Press, London, 2006.

[3] S. Schmidt, M. Breuer, Hybrid LES-URANS methodology for the prediction of non-equilibrium wall-bounded Internal and external flows, Comput. Fluids 96 (2014) 226-252.

[4] L. Marstorp, G. Brethouwer, O. Grundestam, A. Johansson, Explicit algebraic subgrid stress models with application to rotating channel flow, J. Fluid Mech. 639 (2009) 403-432.

[5] B. Chaouat, R. Schiestel, A new partially integrated transport model for subgrid-scale stresses and dissipation rate for turbulent developing flows, Phys. Fluids 17 (065106) (2005) 1-19.

[6] B. Chaouat, R. Schiestel, Progress in subgrid-scale transport modelling for continuous hybrid non-zonal RANS/LES simulations, Int. J. Heat Fluid Fl. 30 (4) (2009) 602-616.

[7] A. Fadai-Ghotbi, C. Friess, R. Manceau, J. Borée, A seamless hybrid RANS-LES model based on transport equations for the subgrid stresses and elliptic blending, Phys. Fluids 22 (2010) 055104.

[8] T. B. Gatski, C. L. Rumsey, R. Manceau, Current trends in modeling research for turbulent aerodynamic flows, Phil. Trans. R. Soc. A 365 (1859) (2007) 2389-2418.

[9] P. Spalart, Detached-eddy simulation, Annu. Rev. Fluid Mech. 41 (2009) 181-202.

[10] A. Fadai-Ghotbi, C. Friess, R. Manceau, T. Gatski, J. Borée, Temporal filtering: a consistent formalism for seamless hybrid RANS-LES modeling in inhomogeneous turbulence, Int. J. Heat Fluid Fl. 31 (3) (2010) 378-389.

[11] S. Kubacki, E. Dick, Simulation of plane impinging jets with $k-\omega$ based hybrid RANS/LES models, Int. J. Heat Fluid Fl. 31 (5) (2010) 862-878.

[12] B. Chaouat, R. Schiestel, Analytical insights into the partially integrated transport modeling method for hybrid Reynold averaged Navier-Stokes equations-large eddy simulations of turbulent flows, Phys. Fluids 24 (2012) 1-34.

[13] B. Geurts, J. Fröhlich, A framework for predicting accuracy limitations in large-eddy simulation, Physics of Fluids 14 (6) (2002) L41L44.

[14] S. Pope, Turbulent Flows, Cambridge University Press, New-York, 2000.

[15] P. Batten, U. Goldberg, S. Chakravarthy, Interfacing statistical turbulence closures with large-eddy simulation, AIAA J. 42 (3) (2004) 485492.

[16] F.-S. Lien, E. Yee, H. Ji, K.-J. Hsieh, Partially resolved numerical simulation and RANS modeling of flow and passive scalar transport in an urban environment, J. Wind Eng. Ind. Aerod. 96 (2008) 1832-1842.

[17] P. Spalart, W.-H. Jou, M. Strelets, S. Allmaras, Comments on the feasibility of LES for wings, and on a hybrid RANS/LES approach, in: C. Liu, Z. Liu (Eds.), First AFOSR International Conference on DNS/LES, 4-8 August, Ruston, LA, Advances in DNS/LES, Greyden Press, Columbus, OH, USA, 1997.

[18] A. Travin, M. Shur, M. Strelets, P. Spalart, Advances in LES of Complex Flows, New York: Kluwer Acad., 2002, Ch. Physical and Numerical Upgrades in the Detached-Eddy Simulation of Complex Turbulent Flows, pp. 239-254.

[19] M. Gritskevich, A. Garbaruk, J. Schütze, F. Menter, Development of DDES and IDDES formulations for the k- $\omega$ shear stress transport model, Flow Turbul. Combust. 88 (3) (2012) 431-449.

[20] R. Schiestel, A. Dejoan, Towards a new partially integrated transport model for coarse grid and unsteady turbulent flow simulations, Theor. Comput. Fluid Dyn. 18 (6) (2005) 443-468. 

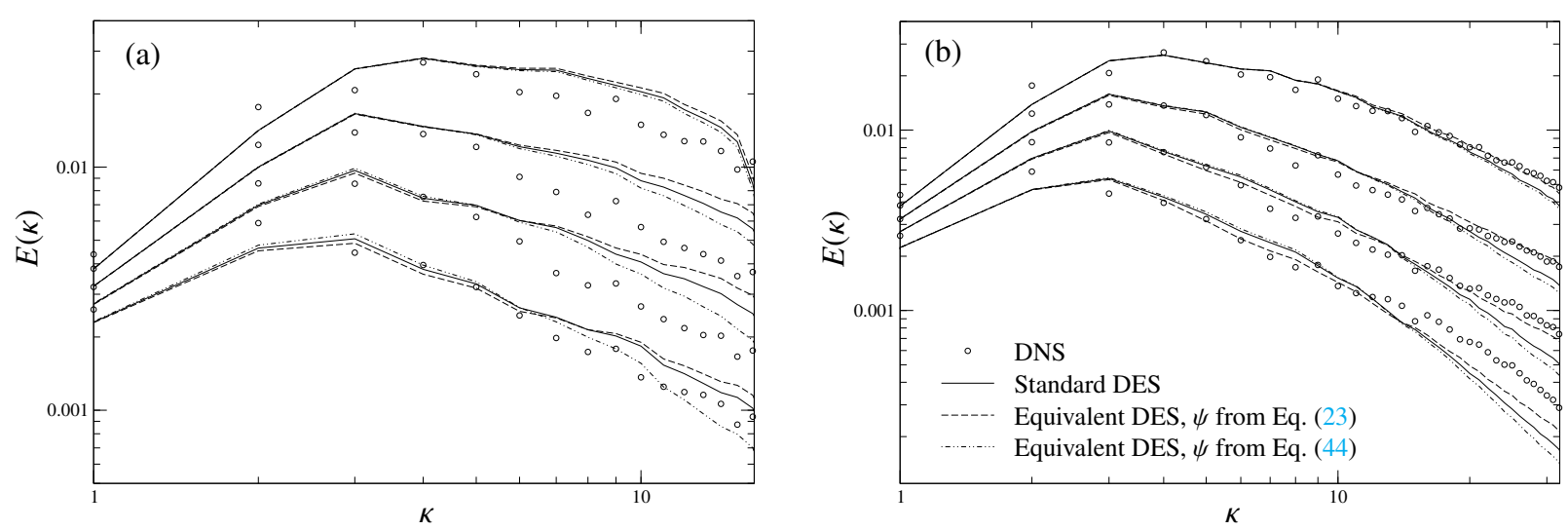

Figure B.10: Decaying isotropic turbulence: comparison of the time evolution of the energy spectra. (a) $32^{3}$ (b) $64^{3}$.

[21] S. S. Girimaji, Partially-averaged Navier-Stokes model for turbulence: A Reynolds-averaged Navier-Stokes to direct numerical simulation bridging method, J. Appl. Mech. 73 (3) (2006) 413-421.

[22] F. R. Menter, Y. Egorov, The scale-adaptive simulation method for unsteady turbulent flow predictions. Part 1: Theory and model description, Flow Turbul. Combust. 85 (1) (2010) 113-138.

[23] L. Davidson, S. Peng, Hybrid LES-RANS modelling: A one-equation SGS model combined with a $k-\omega$ model for predicting recirculating flows, Int. J. Numer. Meth. Fluids 43 (9) (2003) 1003-1018.

[24] B. Chaouat, R. Schiestel, From single-scale turbulence models to multiple-scale and subgrid-scale models by Fourier transform, Theor Comput. Fluid Dyn. 21 (3) (2007) 201-229.

[25] B. Basara, S. Krajnović, S. Girimaji, Z. Pavlovic, Near-Wall Formulation of the Partially Averaged Navier-Stokes Turbulence Model, AIAA J. 49 (12) (2011) 2627-2636.

[26] S. Kubacki, J. Rokicki, E. Dick, Hybrid RANS/LES computations of plane impinging jets with DES and PANS models, Int. J. Heat Fluid Fl. 44 (2013) 596-609.

[27] C. L. Rumsey, B. A. Petterson Reif, T. B. Gatski, Arbitrary steadystate solutions with the $k-\varepsilon$ model, AIAA J. 44 (2006) 1586-1592.

[28] R. D. Moser, J. Kim, N. N. Mansour, Direct numerical simulation of turbulent channel flow up to $R e_{\tau}=590$, Phys. Fluids 11 (4) (1999) 943-945.

[29] M. Breuer, N. Peller, C. Rapp, M. Manhart, Flow over periodic hills numerical and experimental study in a wide range of Reynolds num- bers, Computers and Fluids 38 (2009) 433-457.

[30] R. Manceau, K. Hanjalić, Elliptic blending model: A new near-wall Reynolds-stress turbulence closure, Phys. Fluids 14 (2) (2002) 744 754.

[31] R. Manceau, Recent progress in the development of the Elliptic Blending Reynolds-stress model, Int. J. Heat Fluid Fl.In press.

[32] B. Perot, J. Gadebusch, A self-adapting turbulence model for flow simulation at any mesh resolution, Phys. Fluids 19 (11) (2007) 115105.

[33] S. Bhushan, D. Walters, A dynamic hybrid Reynolds-averaged Navier Stokes-Large eddy simulation modeling framework, Physics of Fluids (1994-present) 24 (1) (2012) 015103.

[34] F. Archambeau, N. Méchitoua, M. Sakiz, Code Saturne: A finite volume code for the computation of turbulent incompressible flows - Industrial applications, Int. J. on Finite Volume, Electronical edition: http://averoes.math.univ-paris13.fr/html ISSN 1634 (0655).

[35] P. Spalart, S. Deck, M. Shur, K. Squires, M. Strelets, A. Travin, A new version of detached-eddy simulation, resistant to ambiguous grid densities, Theor. Comput. Fluid Dyn. 20 (3) (2006) 181-195.

[36] B. Chaouat, Subfilter-scale transport model for hybrid RANS/LES simulations applied to a complex bounded flow, J. Turbul. 11 (51) (2010) $1-30$.

[37] A. Wray, A selection of test cases for the validation of large-eddy simulations of turbulent flows, 1998, AGARD Advisory Report 345. Chapter: HOM02 Decaying Isotropic Turbulence. 